\title{
Simultaneous Estimation of Microphysical Parameters and Atmospheric State with Simulated Radar Data and Ensemble Square Root Kalman Filter. Part I: Sensitivity Analysis and Parameter Identifiability
}

\author{
Minguing Tong And Ming Xue \\ Center for Analysis and Prediction of Storms, and School of Meteorology, University of Oklahoma, Norman, Oklahoma
}

(Manuscript received 31 October 2006, in final form 20 August 2007)

\begin{abstract}
The possibility of estimating fundamental parameters common in single-moment ice microphysics schemes using radar observations is investigated for a model-simulated supercell storm by examining parameter sensitivity and identifiability. These parameters include the intercept parameters for rain, snow, and hail/graupel, and the bulk densities of snow and hail/graupel. These parameters are closely involved in the definition of drop/particle size distributions of microphysical species but often assume highly uncertain specified values.

The sensitivity of model forecast within data assimilation cycles to the parameter values, and the issue of solution uniqueness of the estimation problem, are examined. The ensemble square root filter (EnSRF) is employed for model state estimation. Sensitivity experiments show that the errors in the microphysical parameters have a larger impact on model microphysical fields than on wind fields; radar reflectivity observations are therefore preferred over those of radial velocity for microphysical parameter estimation. The model response time to errors in individual parameters are also investigated. The results suggest that radar data should be used at about 5-min intervals for parameter estimation. The response functions calculated from ensemble mean forecasts for all five individual parameters show concave shapes, with unique minima occurring at or very close to the true values; therefore, true values of these parameters can be retrieved at least in those cases where only one parameter contains error.

The identifiability of multiple parameters together is evaluated from their correlations with forecast reflectivity. Significant levels of correlation are found that can be interpreted physically. As the number of uncertain parameters increases, both the level and the area coverage of significant correlations decrease, implying increased difficulties with multiple-parameter estimation. The details of the estimation procedure and the results of a complete set of estimation experiments are presented in Part II of this paper.
\end{abstract}

\section{Introduction}

The accuracy of numerical weather prediction (NWP) depends very much on the accuracy of the initial state estimation and the accuracy of the prediction model. Various advanced data assimilation techniques have been developed in the recent decades that improve the estimation of model initial conditions. Among these methods are four-dimensional variational data assimilation (4DVAR; Le Dimet and Talagrand 1986; Courtier and Talagrand 1987) and the ensemblebased assimilation methods (Evensen 1994; Evensen

Corresponding author address: Ming Xue, Center for Analysis and Prediction of Storms, National Weather Center, Suite 2500, 120 David L. Boren Blvd., Norman, OK 73072.

E-mail: mxue@ou.edu and van Leeuwen 1996; Burgers et al. 1998; Houtekamer and Mitchell 1998; Anderson 2001; Bishop et al. 2001; Whitaker and Hamill 2002; Evensen 2003; Tippett et al. 2003), which have the advantage of closely involving a numerical model in the data assimilation process. However, errors in the model can directly affect the effectiveness of these data assimilation methods.

For convective-scale NWP, explicit microphysics schemes are used to predict the evolution of clouds and precipitation. Most microphysics schemes use the "bulk" approach of parameterization, in which the particle or drop size distributions (DSDs) are parameterized in functional forms. Often, significant uncertainties exist with the treatment of the microphysical processes and the microphysical parameters. Previous sensitivity studies (e.g., McCumber et al. 1991; Ferrier et al. 1995; 
Gilmore et al. 2004; van den Heever and Cotton 2004) demonstrate that the structure and evolution of simulated convective systems are very sensitive to microphysical parameterizations. Variations in microphysical parameters, such as collection efficiencies, DSD parameters, and particle densities, have profound effects upon the characteristics of precipitation systems and their associated dynamical processes.

Because of many assumptions involved, the microphysical parameterization can be a significant source of model error for convective-scale data assimilation and prediction. Parameter estimation is a common approach to dealing with model error associated with uncertain parameters. The inverse problem of parameter estimation concerns the optimal determination of the parameter by observing the dependent variable(s) collected in the spatial and time domains (Yeh 1986). Various methods have been used for parameter estimation, among which variational parameter estimation with an adjoint model is popular in the literature of meteorology and oceanography (Navon 1998). The ensemble Kalman filter method (hereafter EnKF) has recently been tested successfully for the atmospheric state estimation at the convective scale with simulated (Snyder and Zhang 2003; Zhang et al. 2004; Tong and Xue 2005; Xue et al. 2006) and real (Dowell et al. 2004; Tong 2006) radar data. The results with simulated data, under the perfect model assumption, have been excellent, while the quality of state estimation with real data, when model error inevitably exists, is generally not as good. More recently, Aksoy et al. (2006) used EnKF for the simultaneous estimation of state variables and up to six parameters in a relatively simple two-dimensional sea-breeze model with encouraging success.

In this study, we set out to investigate the ability of the EnKF in correcting the errors in some of the fundamental parameters in model microphysics, where complex process interactions and high nonlinearities usually exist. In the framework of EnKF, parameter estimation is realized by treating the uncertain parameters as independent model variables and using the covariance information sampled from the ensemble to estimate the parameters given available observations (Anderson 2001). This is often referred to as state vector augmentation technique where the model parameters are considered part of the augmented state vector. Model state variables and parameters are estimated simultaneously, through continuous assimilation cycles. The latest estimation will be used for subsequent forecast.

The well-posedness and parameter identifiability are the main issues that are directly related to the possibility of successful parameter estimation, no matter what technique is used. The concept of identifiability addresses the question of whether it is at all possible to obtain unique solutions of the inverse problem for unknown parameters of interest in a model from data collected in the spatial and time domains (Navon 1998). The inverse problem for parameter estimation is often ill-posed (Chavent 1974; Yakowitz and Duckstein 1980). As was reviewed by Yeh (1986), the illposedness is generally characterized by the nonuniqueness and instability of the identified parameters. In the case of nonuniqueness, the estimated value often depends on its initial guess and is not guaranteed to be close to the "true" value. The instability of the inverse solution stems from the fact that small errors in the observations will cause serious errors in the identified parameters. Yakowitz and Duckstein (1980) demonstrated that a small sensitivity of the model output in terms of observations to the change of unknown parameters (parameters to be estimated) implies identification instability. The problem is that a larger difference in the parameter may be manifested by only very small changes in the model output of observed quantities, which may be smaller than anticipated measurement error.

As the first part of this study, we investigate the possibility of retrieving some microphysical parameters with the EnKF method through a detailed sensitivity analysis. The issue of parameter identifiability will be addressed. The results will guide our design of the parameter estimation experiments and also help us understand the estimation results. Such an investigation is necessary because we are dealing with a complex, highly nonlinear system, and the feasibility of estimating DSD-related parameters using a full model and radar observations has not been studied before. The microphysical parameters to be estimated are the intercept parameters of rain, snow, and hail/graupel size distributions, and the bulk densities of hail/graupel and snow. These parameters have been shown by the sensitivity studies referenced earlier to have significant effect on the precipitation processes and dynamics of convective storms. Other model parameters are assumed to be correct.

This paper is organized as follows. In section 2, we briefly discuss bulk microphysics schemes and their limitations, which partly motivate this study. The uncertainties of the chosen microphysical parameters based on previous observational studies will also be discussed. Section 3 briefly describes the numerical model, the simulation configuration for a supercell thunderstorm, and the response function used for sensitivity analysis. The configurations of radar data assimilation via the ensemble square root Kalman filter 

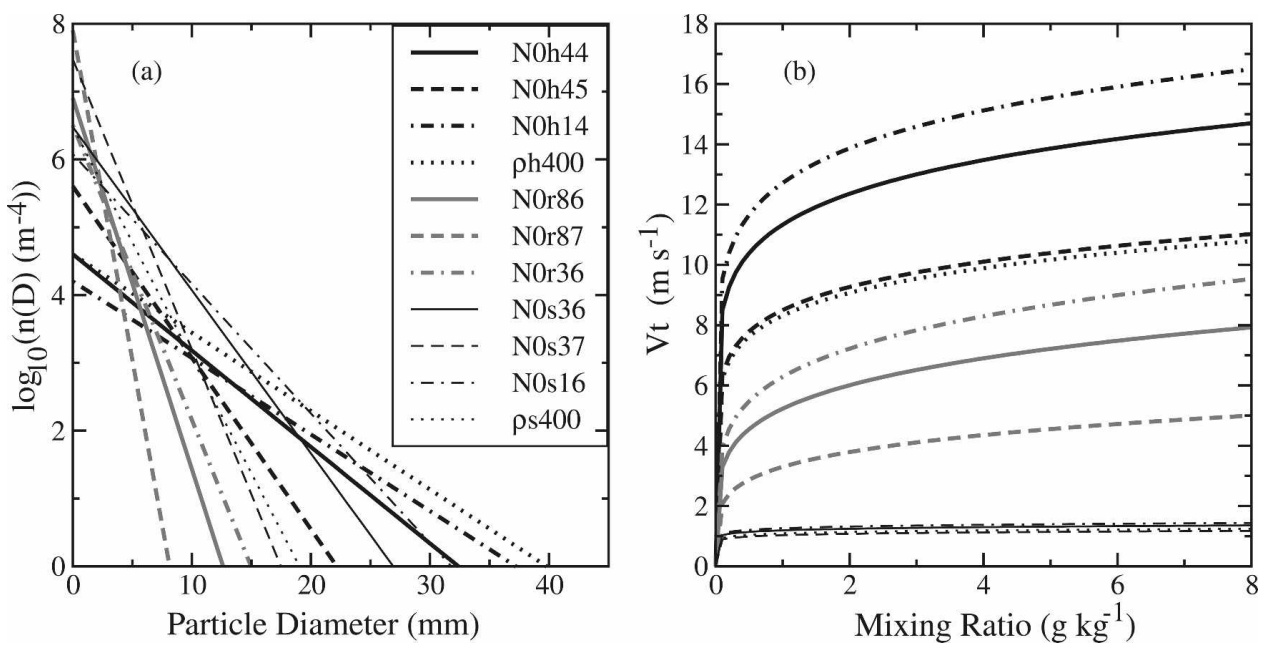

FIG. 1. (a) Number concentration per millimeter diameter size for a mixing ratio of $10 \mathrm{~g} \mathrm{~kg}^{-1}$ and (b) mass-weighted mean terminal velocity of rain (for N0r87: $n_{0 r}=8 \times 10^{7} \mathrm{~m}^{-4}$, N0r86: $n_{0 r}=8 \times 10^{6} \mathrm{~m}^{-4}$, and N0r36: $n_{0 r}=3 \times 10^{6} \mathrm{~m}^{-4}$ ), snow (for N0s37: $n_{0 s}=3 \times 10^{7} \mathrm{~m}^{-4}$, N0s36: $n_{0 s}=3 \times 10^{6} \mathrm{~m}^{-4}$, N0s16: $n_{0 s}=1.19 \times 10^{6} \mathrm{~m}^{-4}$, and $\rho_{s} 400: \rho_{s}=400 \mathrm{~kg} \mathrm{~m}^{-3}$ ), and hail/graupel (for N0h45: $n_{0 h}=4 \times 10^{5} \mathrm{~m}^{-4}$, N0h14: $n_{0 h}=1.59 \times 10^{4} \mathrm{~m}^{-4}$, N0h44: $n_{0 h}=4 \times 10^{4} \mathrm{~m}^{-4}$, and $\left.\rho_{h} 400: \rho_{h}=400 \mathrm{~kg} \mathrm{~m}^{-3}\right)$. The terminal velocities are calculated for an air density of $1.0 \mathrm{~kg} \mathrm{~m}^{-3}$. The default values of the microphysical parameters are $n_{0 r}=8 \times 10^{6} \mathrm{~m}^{-4}, n_{0 s}=3 \times 10^{6} \mathrm{~m}^{-4}, n_{0 h}=4 \times 10^{4} \mathrm{~m}^{-4}, \rho_{h}=913 \mathrm{~kg} \mathrm{~m}^{-3}$, and $\rho_{z}=$ $100 \mathrm{~kg} \mathrm{~m}^{-3}$, unless otherwise indicated by the curve legends.

(EnSRF; Whitaker and Hamill 2002) algorithm, as well as observation operators, are also described. Section 4 discusses the results of sensitivity analysis. The parameter identifiability issue is addressed in section 5. Summary and conclusions are given in section 6 . Results of the parameter estimation experiments are presented in Tong and Xue (2008, hereafter Part II).

\section{Model microphysics}

\section{a. Bulk microphysics schemes}

The microphysics scheme in the Advanced Regional Prediction System (ARPS; Xue et al. 2000, 2001, 2003) model used by this study is a five-class (cloud water, rain, cloud ice, snow, and hail/graupel), single-moment bulk scheme after Lin et al. (1983, hereafter LFO83). The scheme assumes that the exponential DSDs of rain, snow, and hail/graupel have an exponential form:

$$
n_{x}(D)=n_{0 x} \exp \left(-\lambda_{x} D_{x}\right)
$$

where $x$ represents $r$ (rain), s (snow), or $h$ (hail), for particular hydrometeor species. The DSD is assumed monodisperse for nonprecipitating cloud water and cloud ice. In (1), $n_{x}(D) \delta D$ is the number of drops per unit volume between diameters $D$ and $D+\delta D$ and $n_{0 x}$ is the so-called intercept parameter, which is the value of $n_{x}$ for $D=0$. The slope parameter, which is equal to the inverse of the mean size diameter of each distribution, is diagnosed as

$$
\lambda_{x}=\left(\frac{\pi \rho_{x} n_{0 x}}{\rho q_{x}}\right)^{0.25},
$$

where $\rho_{x}$ is the constant particle bulk density, $\rho$ is the air density, and $q_{x}$ is the hydrometeor mixing ratio.

With single-moment bulk microphysics schemes, only one moment of the DSD functions is predicted. In the LFO83 scheme, as well as most other singlemoment schemes, the mixing ratio of each hydrometeor, which is proportional to the third moment of the DSD function, is predicted and the intercept parameter $n_{0 x}$ is a prescribed constant. It can be seen from (1) and (2) that the DSD is a function of two adjustable parameters $n_{0 x}$ and $\rho_{x}$. For model simulations, adjusting these parameters can directly impact the bulk terminal velocity and the number concentration of species (Fig. 1; Fig. 2 of Gilmore et al. 2004), which can result in the change of the trajectories of the hydrometeors within the cloud and the particle growth rates. These changes in the microphysical processes will affect the water budgets within the cloud and hence the latent heating and hydrometeor loading, which in turn lead to the changes of the buoyancy and subsequent updraft and downdraft patterns, hence the storm dynamics.

With the use of prescribed parameters, typical singlemoment microphysics schemes generally cannot ad- 
equately represent convective clouds of different types of precipitation systems. For example, the parameterization of the LFO83 scheme is formulated for the intense continental storms with the presence of high-density hails while the somewhat similar scheme of Rutledge and Hobbs $(1983,1984)$ is more suitable for oceanic systems. The differences come from either the treatment of the microphysical processes and/or the use of different parameters, such as those of hydrometeor density and DSD intercept.

More sophisticated bulk microphysics schemes try to overcome the above limitations by predicting more than one moment of the distribution function and/or dividing the hydrometeors into more categories. By predicting two moments (Ziegler 1985; Murakami 1990; Ferrier 1994; Meyers et al. 1997; Cohard and Pinty 2000) or three moments (Milbrandt and Yau 2005) of the distribution function, the DSD parameters are effectively treated as prognostic variables rather than being prescribed as constants. Straka and Mansell (2005) developed a single-moment bulk microphysics scheme with 10 ice categories, which allows for a range of particle densities and fall velocities for simulating a variety of convective storms with hopefully less need for parameter tuning. Some single-moment (as well as multimoment) schemes assume the gamma distribution (e.g., Milbrandt and Yau 2005), which allows for additional flexibility but also introduces one more free parameter (the shape parameter) that needs to be specified for each species that uses the gamma DSD.

Although sophisticated microphysical schemes are attractive and represent the future direction of convective-scale modeling and NWP, they are expensive and much research is still needed on the treatment of processes involving the additional moments before they can be widely used. The increased number of prognostic variables in the model also poses a larger challenge for state estimation or model initialization. The singlemoment bulk schemes are widely used in current research and operational models; the ultimate goal of our current line of study is therefore to overcome, to the extent possible, the shortcomings of such singlemoment schemes by constraining uncertain microphysical parameters using data, that is, by estimating the parameters as well as the model state variables using radar observations of the convective storms.

\section{b. Uncertainties in the microphysical parameters}

The parameters selected for this study are the intercept parameters of rain, snow, and hail/graupel DSDs, and the bulk densities of snow and hail. Observational and sensitivity studies indicate that the coefficients associated with the formula for hydrometeor fall speeds
TABLE 1. A summary of the uncertainty ranges, defined by the lower bound $\underline{p}_{i}$ and upper bound $\bar{p}_{i}$, and the control (assumed true) values for intercept parameters $n_{0 h}, n_{0 s}, n_{0 r}$, and hail and snow densities $\rho_{h}$ and $\rho_{s}$ used in this study.

\begin{tabular}{cccc}
\hline \hline Parameter $p_{i}$ & $\underline{p}_{i}$ & $\bar{p}_{i}$ & $\begin{array}{c}\text { Control values } \\
\text { of parameters, } p_{\text {ic }}\end{array}$ \\
\hline $\begin{array}{l}\text { Hail/graupel intercept } \\
n_{0 h}\left(\mathrm{~m}^{-4}\right)\end{array}$ & $4 \times 10^{2}$ & $4 \times 10^{6}$ & $4 \times 10^{4}$ \\
$\begin{array}{l}\text { Snow intercept } n_{0 s} \\
\left(\mathrm{~m}^{-4}\right)\end{array}$ & $5 \times 10^{5}$ & $1 \times 10^{8}$ & $3 \times 10^{6}$ \\
$\begin{array}{l}\text { Rain intercept } n_{0 r} \\
\left(\mathrm{~m}^{-4}\right)\end{array}$ & $3 \times 10^{6}$ & $8 \times 10^{7}$ & $8 \times 10^{6}$ \\
$\begin{array}{l}\text { Density of hail/graupel } \\
\rho_{h}\left(\mathrm{~kg} \mathrm{~m}^{-3}\right)\end{array}$ & 400 & 913 & 913 \\
$\begin{array}{l}\text { Density of snow } \rho_{s} \\
\left(\mathrm{~kg} \mathrm{~m}^{-3}\right)\end{array}$ & 20 & 400 & 100 \\
\hline
\end{tabular}

and the collection efficiency parameters are also uncertain and can affect the microphysical processes significantly (e.g., Ferrier et al. 1995; McFarquhar and Black 2004). In this study, we focus on the density and intercept parameters, because they are more fundamental and directly affect a large number of processes in the microphysics parameterization.

As pointed out earlier, with the LFO83 singlemoment bulk microphysics scheme, the intercept parameters and the bulk densities of snow and hail are assumed to be constant in space and time. The default values of the intercept parameters for rain, snow, and hail size distributions in the ARPS model are $8 \times 10^{6}$, $3 \times 10^{6}$, and $4 \times 10^{4} \mathrm{~m}^{-4}$, respectively, following LFO83. The densities of snow and hail are specified to be 100 and $913 \mathrm{~kg} \mathrm{~m}^{-3}$, respectively (see Table 1).

A number of observational studies indicate that the intercept parameters of hydrometeor distributions can vary widely among precipitation systems occurring in different large-scale environments. Also, within the same precipitation system the intercept parameters can vary spatially and with the evolution of the system. The observed hail/graupel intercept parameter, $n_{0 h}$, as reviewed by Gilmore et al. (2004), ranges from $10^{2} \mathrm{~m}^{-4}$ to greater than $10^{8} \mathrm{~m}^{-4}$. Observed snow intercept parameter, $n_{0 s}$, varies from $10^{5}$ to $10^{8} \mathrm{~m}^{-4}$ (Gunn and Marshall 1958; Passarelli 1978; Houze et al. 1979, 1980; Lo and Passarelli 1982; Mitchell 1988; Braham 1990). Joss and Waldvogel (1969) found that $n_{0 r}$ varies between $10^{6}$ and $10^{8} \mathrm{~m}^{-4}$. A number of studies have shown a systematic decrease in $n_{0 r}$ as precipitation changed from convective to stratiform (e.g., Waldvogel 1974; Tokay et al. 1995; Tokay and Short 1996; Cifelli et al. 2000).

In the LFO83 scheme, the term hail is used loosely to represent high-density graupel, ice pellets, frozen rain, and hailstones. The bulk density of hail was found to 
vary between 700 and $900 \mathrm{~kg} \mathrm{~m}^{-3}$ (Pruppacher and Klett 1978). The observed bulk density of wet hail can be as large as $943 \mathrm{~kg} \mathrm{~m}^{-3}$ (El-Magd et al. 2000). The bulk density of graupel ranges from 50 to $890 \mathrm{~kg} \mathrm{~m}^{-3}$ (Pruppacher and Klett 1978). The term snow in the LFO83 scheme is used to represent snow crystals, snowflakes, and low-density graupel particles. Snow density varies greatly from one snow event to the next. The density of freshly fallen snow observed in literature varies from 10 to approximately $350 \mathrm{~kg} \mathrm{~m}^{-3}$. Observed bulk density of snow varies between 10 and $500 \mathrm{~kg} \mathrm{~m}^{-3}$ (Brandes et al. 2007).

All these indicate that there exist great uncertainties with the values of the intercept and density parameters, and assuming same values for all precipitation events can lead to significant errors in the prediction model. Estimating their values for specific events using data is likely to significantly reduce such errors or uncertainties.

\section{Model and experimental settings}

\section{a. The prediction model and truth simulation}

The sensitivity analysis in this part and the parameter estimation in Part II are based on a simulated supercell storm. The configurations of the forecast model and truth simulation are mostly inherited from Tong and Xue (2005, hereafter TX05). Briefly, the ARPS (Xue et al. 2000, 2001, 2003), a fully compressible and nonhydrostatic atmospheric prediction system, is used. The truth simulation is initialized from a modified observed supercell sounding as used in Xue et al. (2001). The LFO83 ice microphysics and 1.5-order turbulent kinetic energy (TKE)-based subgrid-scale turbulence schemes are the only physics options included. The model domain is $64 \mathrm{~km} \times 64 \mathrm{~km} \times 16 \mathrm{~km}$ in size. The horizontal grid spacing is $2 \mathrm{~km}$ and the vertical grid spacing is 0.5 $\mathrm{km}$. A 4-K ellipsoidal thermal bubble centered at $x=$ $48 \mathrm{~km}, y=16 \mathrm{~km}$, and $z=1.5 \mathrm{~km}$, with radii of $10 \mathrm{~km}$ in the $x$ and $y$ directions and $1.5 \mathrm{~km}$ in the $z$ direction is used to initiate the storm. The length of simulation is up to $3 \mathrm{~h}$. The assumed true parameter values, which are the default values of the LFO83 scheme, are used in the truth simulation (Table 1).

In the truth simulation, the initial convective cell strengthens over the first $20 \mathrm{~min}$. The strength of the cell then decreases over the next $30 \mathrm{~min}$ or so, which is associated with the splitting of the cell into two at around $55 \mathrm{~min}$. The right-moving cell tends to dominate the system in the later assimilation period. The updraft reaches a peak value of $44 \mathrm{~m} \mathrm{~s}^{-1}$ at about $90 \mathrm{~min}$. The left-moving cell starts to split again at $95 \mathrm{~min}$. The ini- tial cloud starts to form at about $10 \mathrm{~min}$. Rainwater and ice species first form between 10 and $20 \mathrm{~min}$. More information on the simulated storm can be found in TX05.

\section{b. The EnSRF data assimilation configurations}

The procedure of initializing the ensemble is different from our earlier work in TX05 or Xue et al. (2006, hereafter XTD06). Dowell et al. (2004) and Caya et al. (2005) found that using spatially smoothed perturbations to initialize the ensemble works better than using gridpoint-based random noise. In this study, spatially smoothed perturbations are added to the first guess of the initial condition that is horizontally homogeneous as defined by the modified 20 May 1977 Del City, Oklahoma, supercell sounding (Xue et al. 2001). The spatially smoothed perturbation at grid point $(l, m, n)$ is calculated as

$$
\varepsilon(l, m, n)=E \sum_{(i, j, k) \in S} r(i, j, k) W(i, j, k),
$$

where $r(i, j, k)$ is a random number sampled independently from a normal distribution with zero mean and unit deviation, $W(i, j, k)$ is a $3 \mathrm{D}$ distance-dependent weighting function, and $E$ is a scaling parameter for obtaining the right variance of the perturbation field. A fifth-order correlation function based on Eq. (4.10) of Gaspari and Cohn (1999) is used here for W. It is chosen for its closeness to but lower computational cost than the Gaussian function. The summation is over all grid points that are located within a 3D radius, which is set to $6 \mathrm{~km}$ in this study. This radius is chosen based on the typical decorrelation length scale of background errors of the current type of assimilation problems and is actually the same cutoff radius used by the covariance localization (more on this later).

After the smoothed initial perturbations are obtained, they are rescaled, by determining $E$ in Eq. (3) so that the standard deviation of each perturbation field is equal to a desired value. The standard deviations are, respectively, $2 \mathrm{~m} \mathrm{~s}^{-1}$ for velocity components, $2 \mathrm{~K}$ for perturbation potential temperature, and $0.6 \mathrm{~g} \mathrm{~kg}^{-1}$ for $q_{v}, q_{c}, q_{r}, q_{i}, q_{s}$, and $q_{h}$. These values were obtained through numerical experiments, which gave the right spread and work the best for state estimation. For the mixing ratios of hydrometeors, the perturbations are only added in regions within $6-\mathrm{km}$ horizontal distance from the observed precipitation. They are further limited to the height levels where the particular hydrometeor species are physically expected. Negative values of perturbed mixing ratios are set to zero. The perturbations for the velocity components, potential tempera- 
ture, and specific humidity are added to the entire domain except at the lateral boundaries. Our previous studies (TX05; XTD06) show that spurious cells that may be triggered by added perturbations in nonprecipitation regions can be suppressed by assimilating reflectivity data everywhere.

It is found that by using the spatially smoothed initial perturbations, the ensemble spread of most model variables can grow quickly within the first $5 \mathrm{~min}$ of forecast while the gridpoint-based random perturbations used in TX05 would initially decay significantly in the model, decreasing the spread. Perturbing microphysical fields, which was not done in TX05 or XTD06, also contributes to larger ensemble spread in microphysical variables. Larger initial ensemble spread results in smaller ensemble mean root-mean-square (rms) errors in early assimilation cycles. It is also found that with this new method of initial perturbations, updating model variables that are indirectly related, via observation operator, to reflectivity no longer, as it did in TX05, hurts the analysis during the earlier assimilation cycles. Therefore, in our current configuration, we do not withhold the updating of those indirectly related variables when assimilating reflectivity data.

The same background error covariance localization procedure as used in TX05 and XTD06 is applied here to avoid the influence of unreliable covariances at large distances from the observations. No covariance inflation is applied here, because we found that the difference of the analysis rms errors caused by covariance inflation is smaller than that caused by different realizations of the initial ensemble members. Forty ensemble members are used in the experiments. The initial ensemble forecast starts at $20 \mathrm{~min}$ of the simulated supercell storm. Radar observation volumes are assimilated every $5 \mathrm{~min}$. Both radial velocity and reflectivity, including reflectivity in nonprecipitation regions are assimilated in all assimilation experiments. Other settings are very similar to those of XTD06.

\section{c. Observation operators for radar data}

For observing system simulation experiments (OSSEs), simulated observations are collected from a truth simulation or nature run (see, e.g., Lord et al. 2001). As in XTD06, the radar radial velocity and reflectivity data are sampled from the atmosphere of the truth simulation by using a radar emulator, which is also the observation operator used to assimilate the data. The radial velocity and reflectivity data are assumed to be available from a Weather Surveillance Radar-1988 Doppler (WSR-88D) located at the southwest corner of the model domain, and the radar operates in the standard
WSR-88D precipitation mode, having 14 elevations with the lowest elevation at $0.5^{\circ}$ and the highest at $19.5^{\circ}$. Following XTD06, the simulated observations are assumed to be available on the original radar elevation levels. On each elevation level, it is assumed that the observations are already interpolated from the radar polar coordinates to the Cartesian coordinates. The radar emulator does power-gain-based sampling in the vertical direction to project the data from the model levels to the radar elevation levels [refer to Eqs. (1) and (2) in XTD06].

In this study, some modifications were made in calculating the gridpoint values of radial velocity and reflectivity. In XTD06, it was assumed that the terminal velocity effect had already been removed from the radial velocity data; therefore, the terminal velocity term does not explicitly appear in the equation for radial velocity. Here, this effect is explicitly taken into account, with the fall velocity being calculated from

$$
w_{t}=\frac{w_{t r} Z_{e r}+w_{t s} Z_{e s}+w_{t h} Z_{e h}}{Z_{e r}+Z_{e s}+Z_{e h}},
$$

where $Z_{e r}, Z_{e s}$, and $Z_{e h}$ are the equivalent reflectivity factors $\left(\mathrm{mm}^{6} \mathrm{~m}^{-3}\right)$ of rain, snow, and hail, respectively; $w_{t r}, w_{t s}$, and $w_{t h}$ are the mass-weighted mean terminal velocities of rain, snow, and hail. We employ Eqs. (11), (12), and (13) of LFO83 to calculate these terminal velocities, which are consistent with those in our assimilation model. Including the terminal velocity effect explicitly adds an additional degree of sophistication, and the proper estimation of the terminal velocity in the data assimilation process does rely on a reasonable estimate of the hydrometeor state variables. Because the effect of the terminal velocity is usually small as most elevation angles are small, the nonlinearity in the radial velocity operator is generally weaker than that in the reflectivity operator.

The equivalent reflectivity, $Z_{e}\left(\mathrm{~mm}^{6} \mathrm{~m}^{-3}\right)$ is calculated from the mixing ratios of rainwater, snow, and hail, using the formulas found in TX05, except that the reflectivity equation for dry hail is now included, following Smith et al. (1975). A transition zone from dry to wet hail is defined in the $-2.5^{\circ}$ to $2.5^{\circ} \mathrm{C}$ temperature layer. After the values of $Z_{e}$ on the elevation levels are obtained, they are transformed into the commonly used reflectivity $Z\left[=10 \log _{10}\left(Z_{e}\right)\right]$ in $\mathrm{dB} Z$. If the logarithmically transformed reflectivity is negative, we set it to 0 dBZ. In our data assimilation system, reflectivity $Z$ $(\mathrm{dBZ})$ is directly assimilated.

The five microphysical parameters to be estimated are also involved in the calculation of radar reflectivity and terminal velocity. However, in this study, only the 
errors in the prediction model are considered. The observation operators are therefore assumed to be perfect; that is, the true values of these parameters, denoted by vector $\mathbf{p}^{t}$, are used in the observation operators for all experiments. The observation errors are included by adding Gaussian random errors to the "error free" observations, with the latter obtained by applying the radar emulator to the truth simulation. The standard deviations of the observation errors of radial velocity and reflectivity are assumed to be $1 \mathrm{~m} \mathrm{~s}^{-1}$ and $3 \mathrm{~dB} Z$, respectively.

\section{d. Sensitivity experiments and response function}

In this study, the forward method is used for sensitivity analysis (Crook 1996). For each parameter, we performed a series of sensitivity experiments, within which only the parameter considered is varied within its range of uncertainty while all other model parameters are set to be their assumed true values (Table 1). The true values of the microphysical parameters are used in the control experiments (CNTL).

Suppose $\mathbf{p}=\left(p_{1}, p_{2}, \ldots, p_{n}\right)^{\mathrm{T}}$ is the vector of uncertain microphysical parameters. An admissible set $P_{\text {ad }}$ of $\mathbf{p}$ based on the parameter ranges can be defined as

$$
P_{\mathrm{ad}}=\left\{\mathbf{p} \mid \underline{p}_{i} \leq p_{i} \leq \bar{p}_{i}, \quad i=1,2, \ldots, n\right\},
$$

where $p_{i}$ and $\bar{p}_{i}$ are the lower and upper bounds of the $i$ th parameter. The values of $p_{i}$ and $\bar{p}_{i}$ applied in this study can be found in Table 1 . The admissible set $P_{\text {ad }}$ of $\mathbf{p}$ given in Table 1 may not span all parameter values that might have appeared in the literature, but it covers the major range of variations observed for the five parameters. For hail, for example, we do not use a wider range for $n_{0 h}$, which includes small graupel cases, mainly because the LFO83 scheme is designed for hail. Parameters associated with fall speed and collection efficiencies are expected to change, if the DSD has a large change. A wider admissible set $P_{\text {ad }}$ can be used, if the uncertainties in other microphysical parameters are appropriately taken into account, which needs to be considered in future work.

The parameter estimation problem consists of finding an estimated value $\hat{\mathbf{p}}$ of $\mathbf{p}$ from information contained in the observations, the parameter-toobservation mapping, and the prior information about the parameters. The problem can often be constructed as finding $\hat{\mathbf{p}} \in P_{\mathrm{ad}}$, such that $J(\hat{\mathbf{p}})=\min J(\mathbf{p}), \forall \mathbf{p} \in P_{\mathrm{ad}}$. Here $J(\mathbf{p})$ is an output criterion that measures the difference between the observations and the model output of observations. Therefore, in this study, we are especially interested in the sensitivity of the model output, in the form of observations, to the microphysical parameters.

We define a response function for the sensitivity analysis as

$$
J_{y}(\mathbf{p})=\frac{1}{\sigma_{y}^{2}} \sum_{m=1}^{M}\left[y_{m}(\mathbf{p})-y_{m}^{o}\right]^{2},
$$

where $y_{m}(\mathbf{p})$ and $y_{m}^{o}$ are, respectively, the model solution in the form of observation and the corresponding observation, and $\sigma_{y}$ is the standard deviation of the estimated observation errors. The observations in the current case contain the simulated radial velocity $V_{r}$ and reflectivity $Z ; M$ is the total number of data points where reflectivity is greater than $0 \mathrm{~dB} Z$. This response function has essentially the same form as the observation term in the typical three-dimensional variational data assimilation (3DVAR) cost function, under the assumption that observation errors are uncorrelated. When $y$ is derived from the model forecast, (6) measures the departure of the model forecast from the observations, and this departure can be due to both initial condition (state estimation) error and model (microphysical parameter) error.

For parameter estimation with the EnSRF method, state variables and uncertain parameters are estimated simultaneously in continuous data assimilation cycles. The possibility of estimating an uncertain parameter depends on the model's response to the parameter error within the data assimilation cycles. Therefore, the model forecasts during the data assimilation will be used to evaluate the response function.

\section{Results of experiments}

The ensemble-based forecast and assimilation results are sensitive to the realization of the initial ensemble perturbations. To increase the robustness of the results to be presented in this paper, we performed five parallel sets of experiments, with the only difference being the initialization of ensembles. All the response functions shown in this paper are averaged over the five parallel sets of experiments. The results of the CNTL data assimilation experiments are first presented to show the behavior of the EnKF state estimation without parameter error.

Figure 2 shows the rms errors of the ensemble mean forecasts and analyses of the five CNTL experiments, together with mean of the ensemble spread from five experiments (thick curves). All analyses tend to converge at about $70 \mathrm{~min}$. The agreement among the five experiments indicates the robustness of our data assimilation. We note here that the larger initial ensemble 

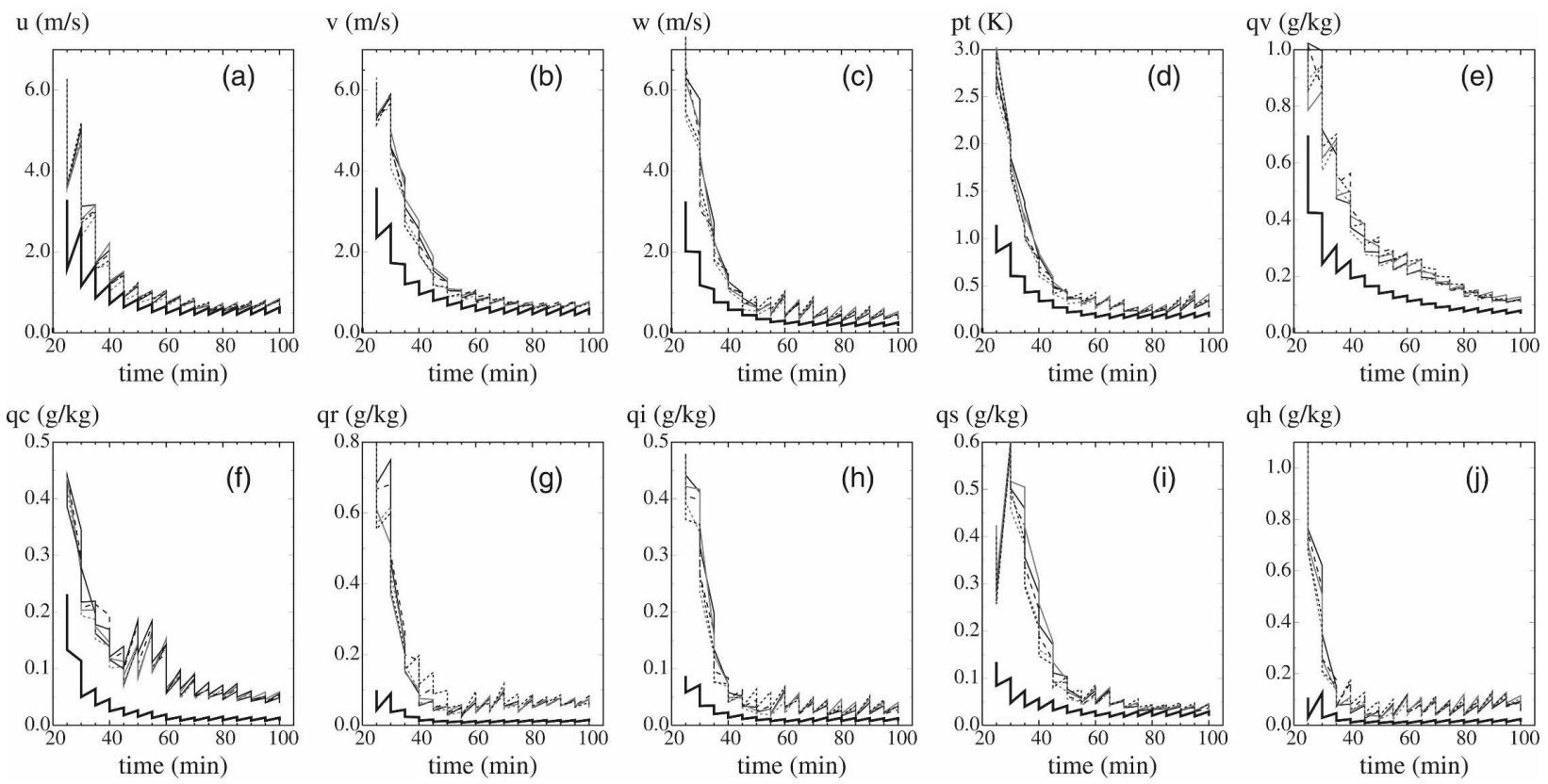

FIG. 2. The rmse of the ensemble mean forecasts and analyses (thin curves of different line patterns) averaged over points at which the true reflectivity is greater than $10 \mathrm{dBZ}$ for (a) $u$, (b) $v$, (c) $w$, (d) $\theta^{\prime}$, (e) $q_{v}$, (f) $q_{c}$, (g) $q_{r}$, (h) $q_{i}$, (i) $q_{s}$, and (j) $q_{h}$, from five CNTL data assimilation experiments, with difference in the realization of the initial ensemble. The analysis and forecast ensemble spread averaged over the five experiments is indicated by the thick black curves.

spread due to the use of smoothed initial perturbations and to the addition of perturbations to moisture and microphysical variables results in smaller errors during the early (first five to six) assimilation cycles but similar errors in later cycles (cf. Fig. 9 of TX05).

\section{a. Sensitivity as revealed by response function}

We first performed a series of assimilation sensitivity experiments for each individual parameter. We sampled several possible values of the parameter within $P_{\text {ad }}$, which usually differ from the assumed true value. Such "wrong" values were used within the forecast model that is used in the EnSRF data assimilation experiments. Other parameters that are not considered assumed their true values. The ensemble mean forecast at the end of each 5-min analysis cycle and before each analysis is used to calculate the response function $J_{y}(\mathbf{p})$, and these response functions are averaged over 16 cycles that span 20 through $100 \mathrm{~min}$ of model time, and over five sets of parallel experiments that differ only in the realization of the initial state variable ensemble perturbations. In another word, the CNTL assimilation experiments are repeated, each time one of microphysical parameters is set to a wrong value. The forecast response during the assimilation cycles to the parameter error is examined.

Figure 3 shows the variations of the response func- tions averaged over the data assimilation cycles against the deviation of the parameters from their true values. The microphysical parameters are expressed in logarithmic form since most of them can vary by more than an order of magnitude. The symbols on each curve represent parameter values sampled from $P_{\text {ad }}$. To facilitate the comparison, $\Delta J_{y}(\mathbf{p})=J_{y}(\mathbf{p})-J_{y, c}\left(\mathbf{p}^{t}\right)$ are presented in Figs. 3a and 3b. Here $J_{y, c}\left(\mathbf{p}^{t}\right)$ is the response function calculated from the CNTL assimilation experiment. The response functions $J_{y}(\mathbf{p})$ within the parameter deviation range of $[-10,10]$ are presented in Figs. $3 \mathrm{c}$ and 3d. It is stated that $V_{r}$ or $Z$ is more sensitive to one parameter than the other if the same amount of change in the parameter value causes more change in the response function. As can be seen from Figs. $3 a$ and $3 b$, model reflectivity shows a much stronger sensitivity to all five parameters than model radial velocity. When the deviations of the parameters in logarithmic form are greater than 2 , that is, $\left|\Delta 10 \log _{10}\left(p_{i}\right)\right|>2, \Delta J_{Z}(\mathbf{p})$ are generally more than two times larger than $\Delta J_{V_{r}}(\mathbf{p})$. This is not surprising because the microphysical fields are more directly affected by microphysical parameterization than the velocity field. The larger sensitivity of $Z$ to the microphysical parameters suggests that $Z$ data should be more useful for microphysical parameter estimation. With respect to the three intercept parameters, reflectivity shows larger sensitivity to the inter- 

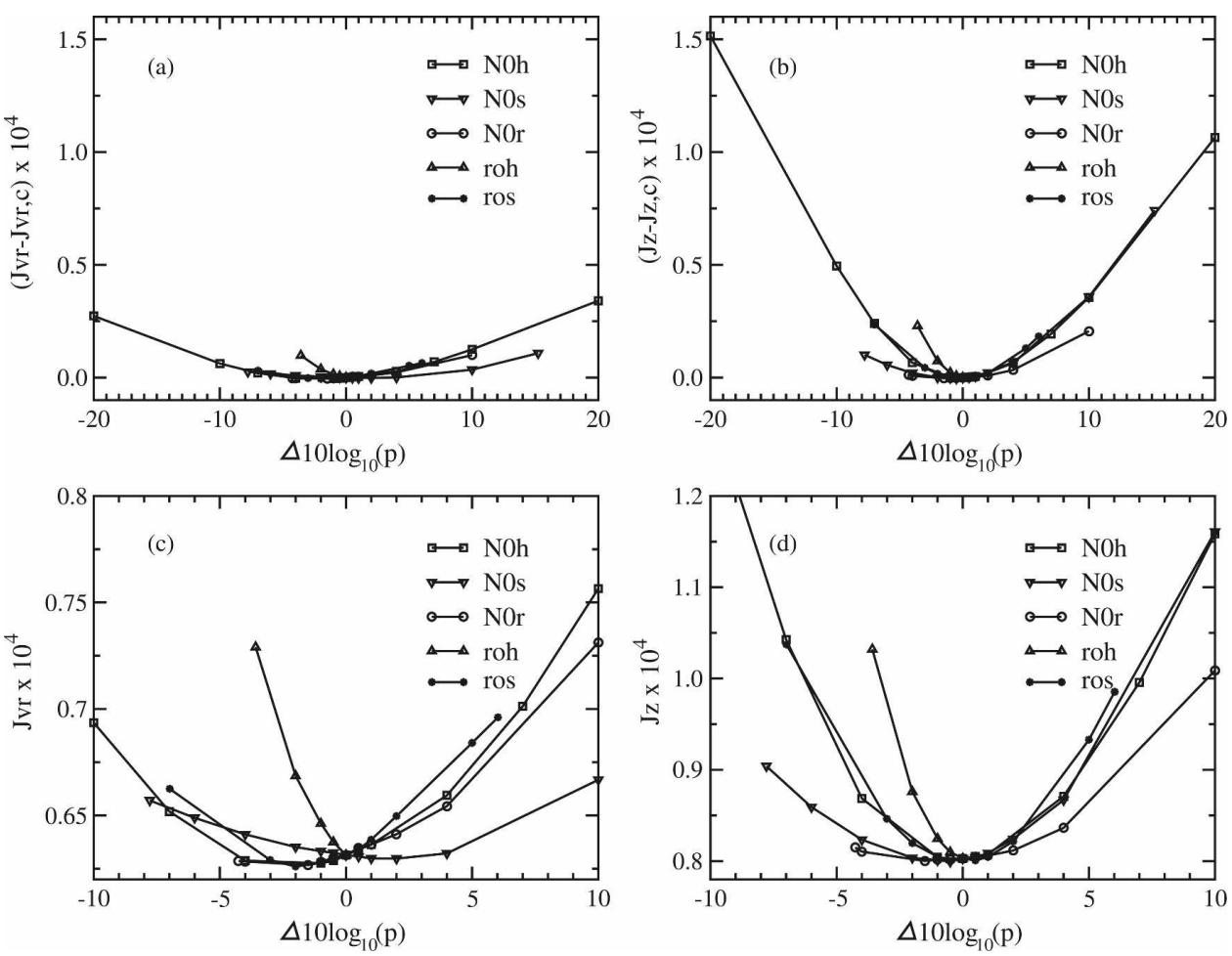

FIG. 3. The variations of the response function (a), (b) $\Delta J_{y}(\mathbf{p})=J_{y}(\mathbf{p})-J_{y, c}\left(\mathbf{p}^{t}\right)$ and (c), (d) $J_{y}(\mathbf{p})$ for (left) radial velocity $V_{r}$ and (right) reflectivity $Z$ against $\Delta 10 \log _{10}(\mathbf{p})=10 \log _{10}(\mathbf{p})-10 \log _{10}\left(\mathbf{p}_{c}\right)$, the logarithmic-form deviation of the parameters from their true values. The response functions were calculated from the ensemble mean forecast at the end of each 5-min analysis cycle and before each analysis and averaged over the 16 assimilation cycles and over five parallel runs with different realizations of initial state variable perturbations. Here $J_{y, c}\left(\mathbf{p}^{t}\right)$ represents the response function calculated from the control (parameter error free) data assimilation experiment.

cept of hail and snow than to the intercept of rain (Fig. 3d). Model reflectivity is more sensitive to $\rho_{h}$ than to $\rho_{s}$ when $\Delta 10 \log _{10}\left(p_{i}\right)<0$.

\section{b. Model response time to errors in microphysical parameters}

An issue associated with parameter estimation is the time scale of model response to the parameter error. If model response is too slow, it will take a long time for the estimation system to "detect" and correct the parameter error, even if there are frequent observations. In the case that the parameter is time varying, successful parameter estimation needs to occur before significant change in the parameter value occurs.

To investigate model response time to microphysical parameter errors, forecast sensitivity experiments are performed, with the forecasts launched from the ensemble mean analyses of CNTL assimilation experiments, at 5-min intervals from 35 through $80 \mathrm{~min}$. All forecasts are run for $40 \mathrm{~min}$ (no additional data assimilation occurs after the forecast is initialized from the ensemble mean analysis). For each starting time (e.g., $35 \mathrm{~min}$ ), one CNTL forecast and five pairs of sensitivity forecasts are performed for each CNTL assimilation experiment. In each of the sensitivity experiments, one of the microphysical parameter is perturbed, with the perturbation values given in Table 2 . Each parameter is perturbed by a larger positive or a smaller negative value, except for hail density, whose true value is equal to its upper bound of $913 \mathrm{~kg} \mathrm{~m}^{-3}$, and therefore cannot have positive perturbation. For hail density, a larger negative perturbation is used. These large and small values form pairs in the total of 10 experiments corresponding to each CNTL assimilation experiment. The perturbed values are chosen to be within the admissible set, and to facilitate the comparison of sensitivities to different parameters we choose perturbations of the same magnitude for the intercept parameters. These experiments are repeated five times, corresponding to the five CNTL assimilation experiments; average results will be presented for robustness of results.

The response function for reflectivity is calculated for 
TABLE 2. List of the parameters values, $p_{i}$, and their logarithmical deviations from the true values, $\Delta 10 \log _{10}\left(p_{i}\right)$, in the sensitivity experiments. The parameter that is changed from its true value is listed for the corresponding sensitivity experiment while other parameters used the true values.

\begin{tabular}{|c|c|c|c|c|c|c|}
\hline \multirow[b]{2}{*}{ Parameter } & \multicolumn{3}{|c|}{ Larger deviation } & \multicolumn{3}{|c|}{ Smaller deviation } \\
\hline & Experiment & $p_{i}$ & $\Delta 10 \log _{10}\left(p_{i}\right)$ & Experiment & $p_{i}$ & $\Delta 10 \log _{10}\left(p_{i}\right)$ \\
\hline$n_{0 h}\left(\mathrm{~m}^{-4}\right)$ & N0h45 & $4 \times 10^{5}$ & 10 & N0h14 & $1.59 \times 10^{4}$ & -4 \\
\hline$n_{0 s}\left(\mathrm{~m}^{-4}\right)$ & N0s37 & $3 \times 10^{7}$ & 10 & N0s16 & $1.19 \times 10^{6}$ & -4 \\
\hline$n_{0 r}\left(\mathrm{~m}^{-4}\right)$ & N0r87 & $8 \times 10^{7}$ & 10 & N0r36 & $3.18 \times 10^{6}$ & -4 \\
\hline$\rho_{h}\left(\mathrm{~kg} \mathrm{~m}^{-3}\right)$ & $\rho_{h} 400$ & 400 & -3.58 & $\rho_{h} 576$ & 576 & -2 \\
\hline$\rho_{s}\left(\mathrm{~kg} \mathrm{~m}^{-3}\right)$ & $\rho_{s} 400$ & 400 & 6 & $\rho_{s} 63$ & 63 & -2 \\
\hline
\end{tabular}

each of the forecast experiments. We use the ratio of the response function of the sensitivity forecast to that of the corresponding CNTL forecast as a measure of the model response to the parameter error relative to the model response to initial condition error (since the estimated state used to start each forecast is imperfect). These ratios are calculated for all pairs of forecasts and averaged over all starting times (i.e., $35-80 \mathrm{~min}$ at 5-min intervals) and over five sets corresponding to the five CNTL experiments. The average ratios are plotted in Fig. 4, for different parameter perturbations, for response functions calculated against error-free and error-containing observations, respectively. In the former case, we are verifying the model forecast against the truth, in terms of the reflectivity, while the latter case reflects how the data assimilation system sees the response.

The response function, which is a measure of the forecast error in terms of $Z$, increases with time during the forecast due to the initial condition error in the CNTL forecasts and due to both initial condition and model errors in the sensitivity forecasts. The response function ratios generally increase within the first 5 to 15 min of forecast, indicating that the forecast error in the presence of parameter error grows faster than that in CNTL during the period. Because eventually the errors
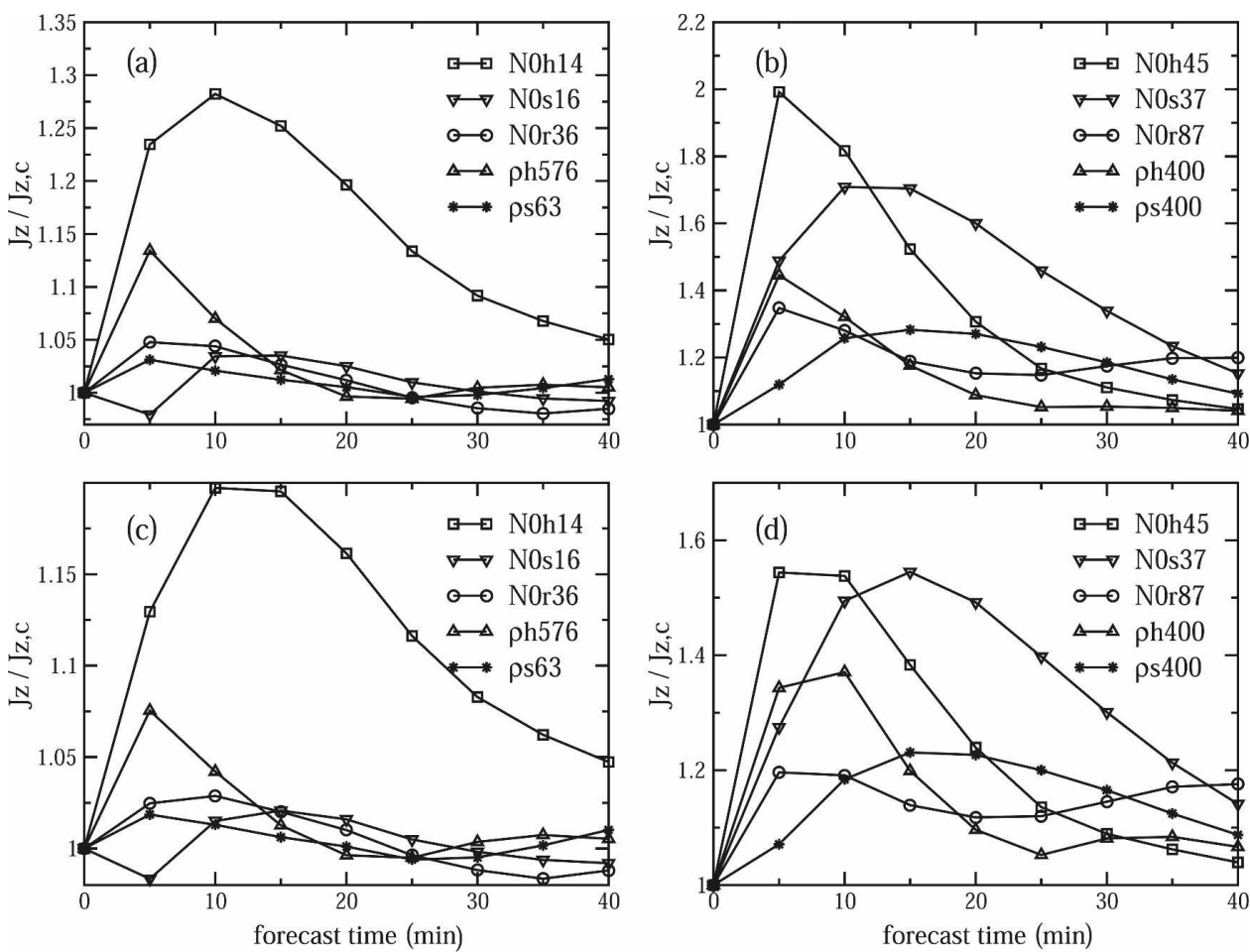

FIG. 4. Time evolution of the ratios of the response functions for $Z$, of forecast sensitivity experiments (cf. Table 2), to that of the CNTL forecast experiment, $J_{z}(\mathbf{p}) / J_{z c}(\mathbf{p})$. The response functions are calculated against (a), (b) error-free reflectivity data and against (c), (d) error-containing reflectivity data. Smaller parameter errors are used in (a) and (c) and larger ones are used in (b) and (d). 
in both CNTL and sensitivity forecasts saturate and because of possible nonlinear effects, the response function ratios do not continue to increase with time. In fact, they are found to peak between 5 and 15 min after the initial time for most parameters. After the peak, the parameter-error-induced response function difference starts to decrease in relative magnitude. In an effort to choose a more objective measure of the model response time to the parameter error, we choose to use the length of time that the response function ratio peaks as the time scale of parameter response. According to these experiments, such time scales are rather short, between 5 and $15 \mathrm{~min}$ for these parameters. Given these time scales, it appears necessary that we perform our parameter estimation using observations every 5 min. Such assimilation frequency appears to match the time scales of the model response well. This does not mean that successful parameter estimation will necessarily occur at this time scale, however. From Fig. 4, we see that the ratios calculated using error-free and errorcontaining data show similar trends.

Among the three intercept parameters, the forecast reflectivity responds fastest to the error in that of hail according to Fig. 4. When the three intercept parameters have the same amount of negative deviations from the truth, the reflectivity forecast is much more sensitive to $n_{o h}$ than to the other two intercept parameters (Figs. 4a,c). The forecast reflectivity also responds faster to the error in $\rho_{h}$ than in $\rho_{s}$. These results suggest that the hail intercept and density parameters are easier to estimate than other parameters.

\section{c. Sensitivity of hydrometeor distributions to microphysical parameters}

To better understand how the model responds in terms of reflectivity to the changes in the microphysical parameters in the presence of parameter error, we now examine the variations in microphysical fields as a result of parameter changes. The impact of the microphysical parameters on storm dynamics and on surface precipitation is not the focus of this study, however. Some of the impacts have been discussed by Gilmore et al. (2004) and van den Heever and Cotton (2004). In this study, we will focus on the impacts of these parameters on microphysical fields as they are directly related to the issue of microphysical parameter sensitivity and identifiability using reflectivity data.

Figures $5 \mathrm{~b}-\mathrm{f}$ show the difference in precipitating hydrometeor mixing ratios $q_{r}, q_{s}$, and $q_{h}$ between the sensitivity forecast experiments N0h43 $\left(n_{0 h}=4 \times 10^{3} \mathrm{~m}^{-4}\right)$, N0s37, N0r87, $\rho_{h} 400$, and $\rho_{s} 400$ (Table 2) and the CNTL forecast experiments. All these forecast experiments were initialized from the ensemble mean analysis of a
CNTL data assimilation experiment at $60 \mathrm{~min}$. The results of 10-min forecast are presented. Because the reflectivity formulation is a function of $q_{r}, q_{s}$, and $q_{h}$, only, the sensitivity in reflectivity results mainly from the sensitivities in these three categories. The corresponding differences in reflectivity are shown in Figs. 6b-f. Reversed patterns in hydrometeors and reflectivity difference fields were found when the parameters were perturbed in opposite directions (not shown).

Figures $5 \mathrm{~b}$ and $5 \mathrm{e}$ show that decreasing $n_{0 h}\left(\rho_{h}\right)$ results in less (more) hail aloft within the convective region and in the anvil and more (less) hail falling to the ground. This mainly results from less (more) collection of cloud water and cloud ice by hail within the convective region in the middle and upper levels due to the lower (higher) number concentration and larger (smaller) fall speed of hails associated with a smaller $n_{0 h}\left(\rho_{h}\right)$ (Fig. 1). The reflectivity difference in Figs. 6b and $6 \mathrm{e}$ mainly results from the change of the production of $q_{h}$ in the corresponding regions. The variations in the hydrometeors due to the changes in the hail parameters are consistent with what were found by Gilmore et al. (2004).

Figures $5 \mathrm{c}$ and $5 \mathrm{f}$ show that increasing $n_{0 s}$ leads to more production of snow, while increasing $\rho_{s}$ results in less production of snow. By examining the source and sink terms of the hydrometeors, we found that a higher number concentration of snow due to a larger $n_{0 s}$ (Fig. 1a) results in more accretion of cloud water and cloud ice by snow, therefore an increased amount of $q_{s}$ (Fig. 5c). Because of the scavenging of $q_{c}$ and $q_{i}$ by $q_{s}$, less $q_{h}$ is produced by accretion of cloud water and cloud ice. Contrarily, a lower concentration of snow due to the increase in $\rho_{s}$ (Fig. 1a) reduces the accretion of cloud water and cloud ice by snow; therefore less $q_{s}$ is produced (Fig. 5f). Increasing $\rho_{s}$ also results in less $q_{h}$. This mainly results from the reduction in the accretion of snow by hail and the accretion of snow by rain, which are two of the production terms for hail. Although the accretion of cloud water and cloud ice by hail increases, the increase in these two terms cannot completely compensate the reduction in the two hail production terms discussed above. The reduction of $q_{h}$ in both cases leads to weaker reflectivity in the convective and anvil precipitation regions at the middle levels (Figs. 6c,f).

Finally, we can see from Fig. 5d that increasing $n_{0 r}$ enhances $q_{r}$ and $q_{h}$ within the convective region Fig. 5d. Rainwater also falls to a broader area around the convection region. Therefore, more reflectivity increments can be seen within and around the convective region at low levels (Fig. 6d). The most sensitive region for reflectivity is actually at the low-level anvil precipitation region. Larger $n_{0 r}$ leads to much weaker reflectivity 

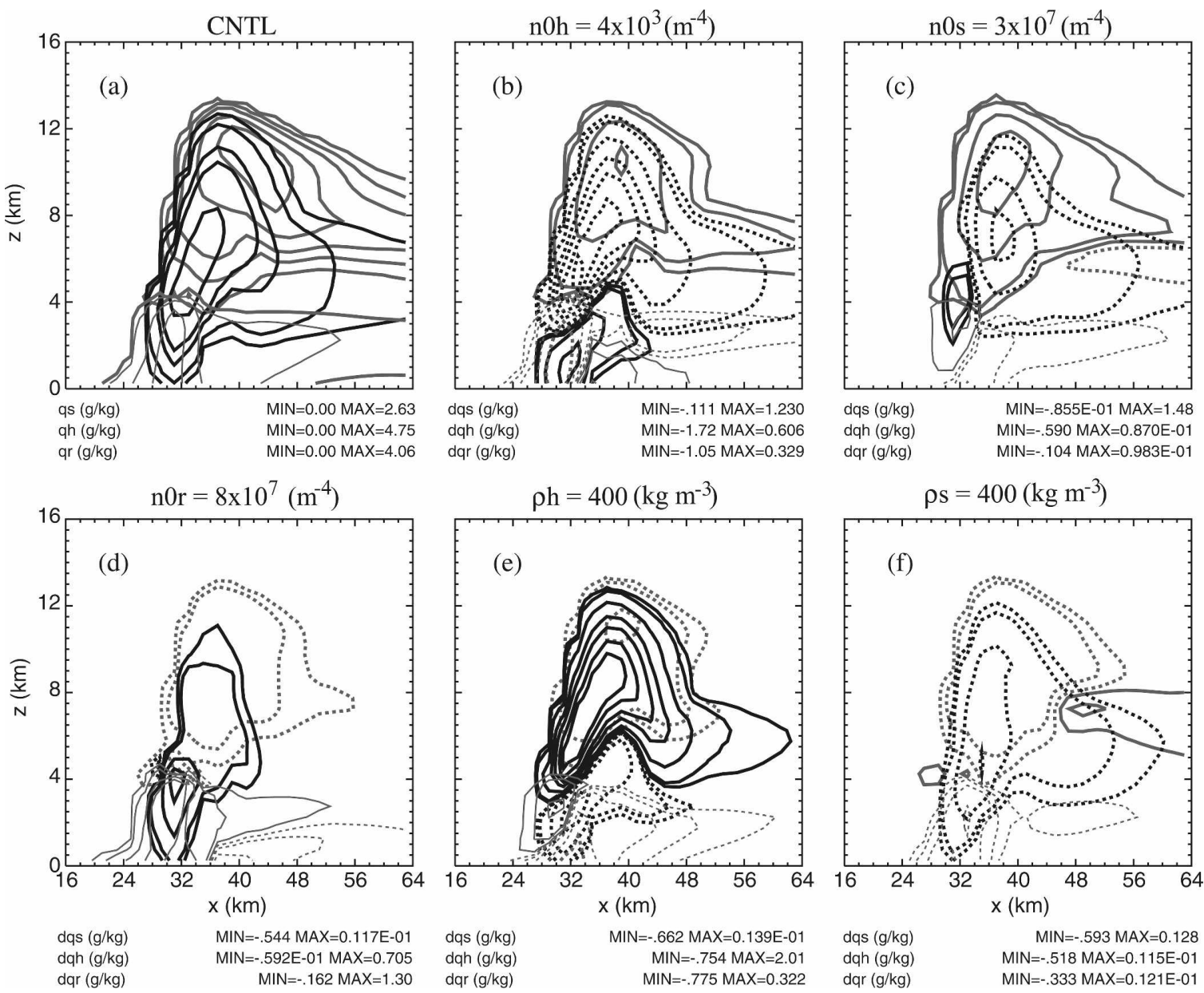

$$
\begin{array}{r}
M I N=-.593 \text { MAX }=0.128 \\
M I N=-.518 \text { MAX }=0.115 E-01 \\
M I N=-.333 \text { MAX }=0.121 E-01
\end{array}
$$

FIG. 5. Vertical cross sections of (a) mixing ratios $\left(\mathrm{g} \mathrm{kg}^{-1}\right): q_{r}$ at intervals of $0.025,0.1,0.8,2.5$, and 5.0 (thin gray); $q_{h}$ at intervals of $0.025,0.1,0.5,1.0,3.0$, and 5.0 (thick black); and $q_{s}$ at intervals of $0.05,0.2,0.5,1.2$, and 2.0 (thick gray) for CNTL forecast experiment; and mixing ratio differences [ $\mathrm{g} \mathrm{kg}^{-1}$; sensitivity experiment - CNTL; solid (dashed) contours represent positive (negative) values] for $q_{r}$ at intervals of $-0.6,-0.3,-0.1,-0.04,0.015,0.015,0.08,0.3$, and $0.6 ; q_{h}$ at intervals of $-1.5,-1.2,-0.8,-0.5,-0.2,-0.05,-0.025$, $0.025,0.05,0.2,0.5,0.8,1.2$, and 1.5 (thick black); and $q_{s}$ at intervals of $-1.5,-1.2,-0.6,-0.1,0.025,0.025,0.1,0.6,1.2$, and 1.5 (thick gray) for forecast experiment (b) N0h43, (c) N0s37, (d) N0r87, (e) $\rho_{h} 400$, and (f) $\rho_{s} 400$ through the maximum updraft at $t=70$ min. The forecast experiments were initialized from the ensemble mean analysis of the CNTL experiment at $t=60$ min. Irregular contour intervals are used to facilitate the comparison with the reflectivity in Fig. 6, which is a log function of the mixing ratios.

there. This is because less $q_{r}$ is found below $2 \mathrm{~km}$ in that region (Fig. 5d). Both the increase in the number of small raindrops and the decrease in the terminal fall speed as a result of increasing $n_{0 r}$ (Fig. 1) enhance the evaporation for raindrops at the low levels, which leads to less rain reaching the ground below the anvil.

Comparing Fig. 6b with Fig. 6e, we can see that the model responds to the changes in hail intercept parameter and density in opposite directions in terms of reflectivity. To see whether the model responses to the errors in the two hail parameters can cancel each other, we performed another forecast experiment, in which the two parameters were perturbed in the same way as in the experiments shown in Figs. $6 \mathrm{~b}$ and 6e, although simultaneously instead of individually. It can be seen from Fig. 7a that the model responses to their errors indeed cancel each other in a large part of the storm. Significant reflectivity sensitivities can only be seen in the region below anvil close to the eastern boundary of the domain. Such reflectivity sensitivities are also shown in Figs. 6c and 6f. We further perturbed $n_{0 s}$ in addition to $n_{0 h}$ and $\rho_{h}$. Decreasing $n_{0 s}$ alone leads to reversed model response compared to that shown in Fig. 6c, that is, to positive reflectivity increments in the anvil precipitation region (not shown). Figure $7 \mathrm{~b}$ shows that the reflectivity sensitivities in Fig. 7a can be further reduced by decreasing $n_{0 s}$. In other words, the model response to the error in $n_{0 s}$ can further cancel the model 

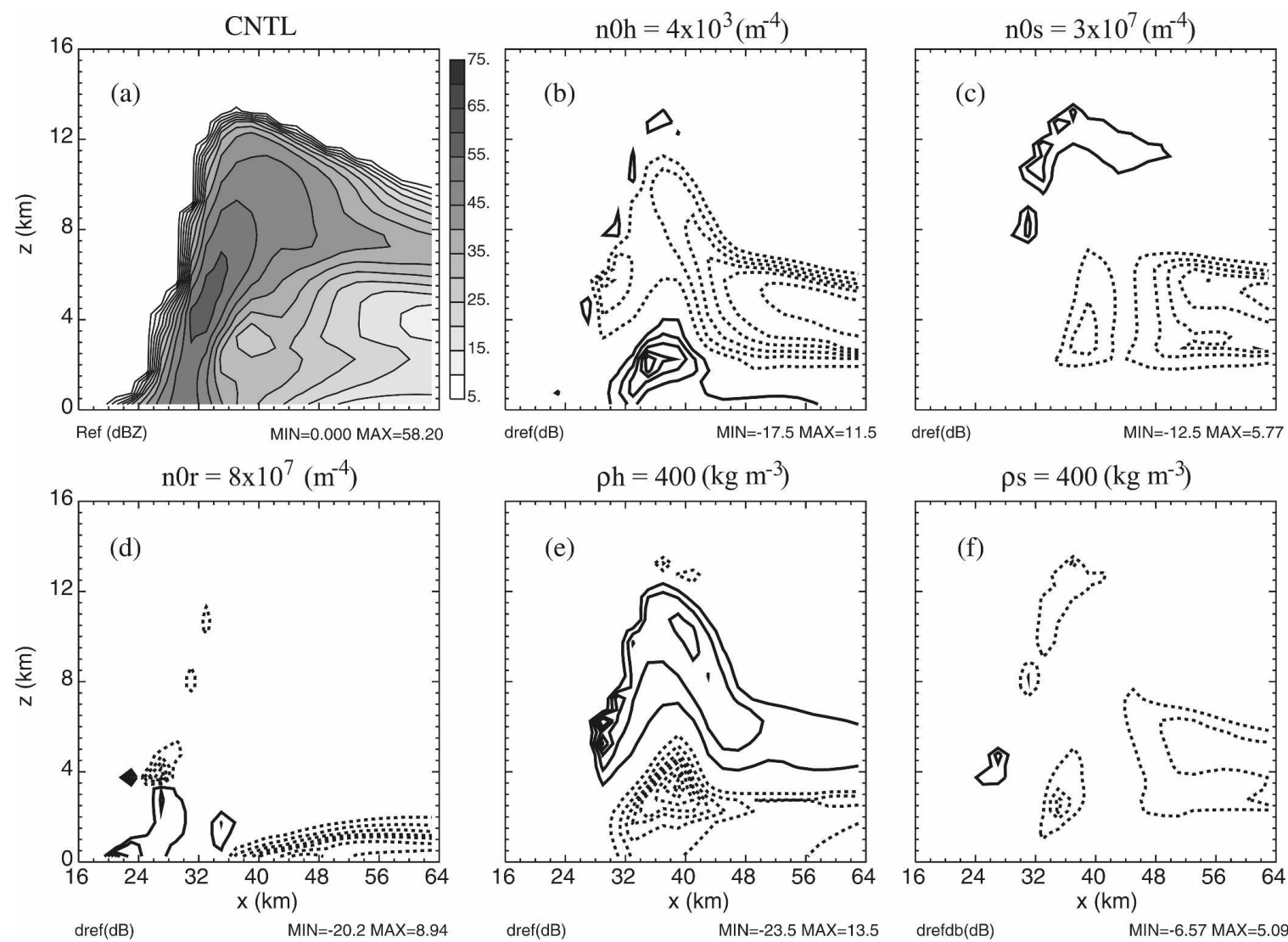

FIG. 6. Vertical cross sections of (a) reflectivity $(\mathrm{dBZ})$ at intervals of $5 \mathrm{dBZ}$ for CNTL forecast experiment, and reflectivity differences (sensitivity experiment - CNTL) at intervals of $\pm 26, \pm 22, \pm 18, \pm 14, \pm 10, \pm 8, \pm 6, \pm 4, \pm 2$, and $0 \mathrm{~dB} Z$ [solid (dashed) contours represent positive (negative) values] through the maximum updraft at $t=70$ min for forecast experiments (b) N0h43, (c) N0s37, (d) N0r87, (e) $\rho_{h} 400$, and (f) $\rho_{s} 400$.

responses to the errors in $n_{0 h}$ and $\rho_{h}$. It is possible that when multiple microphysical parameters contain errors, the errors can combine to give a model reflectivity field that is very close to the true reflectivity, making the simultaneous estimation of these parameters from reflectivity data alone difficult.

\section{Parameter identifiability}

\section{a. Parameter identifiability as revealed by the response function}

An important issue associated with parameter estimation is the parameter identifiability. Various definitions of parameter identifiability can be found in the literature (Kitamura and Nakagiri 1977; Chavent 1979; Sun and Yeh 1990). A definition suitable for the estimation process using the output least squares error criterion was given by Chavent (1979). A parameter is said to be least squares identifiable if the least squares performance function for identifying the parameter has a unique minimum in a given region and if the minimi- zation is continuously dependent on the measurement errors.

The response function defined by (6) is actually the performance function that is to be minimized if the inverse problem is solved by using the output least squares error criterion. As shown in Fig. 3d, the response functions of $Z$ against the variations in the five parameters all have a concave shape, and there is a unique minimum for each case. This is an indication of a unique mapping between the parameters and the model solution in terms of radar reflectivity observations, even though the microphysical process and the observation operators are highly nonlinear.

The minima of the response functions for reflectivity, $J_{Z}$, are not always located exactly at the zero deviation point, but are always close to it (Fig. 3d). The concave shape of the response functions and their single minimum indicate a high probability of finding the true value by using reflectivity data. Although the response functions for radial velocity, $J_{V_{r}}$, also show a concave shape, the minima are located farther away from the 

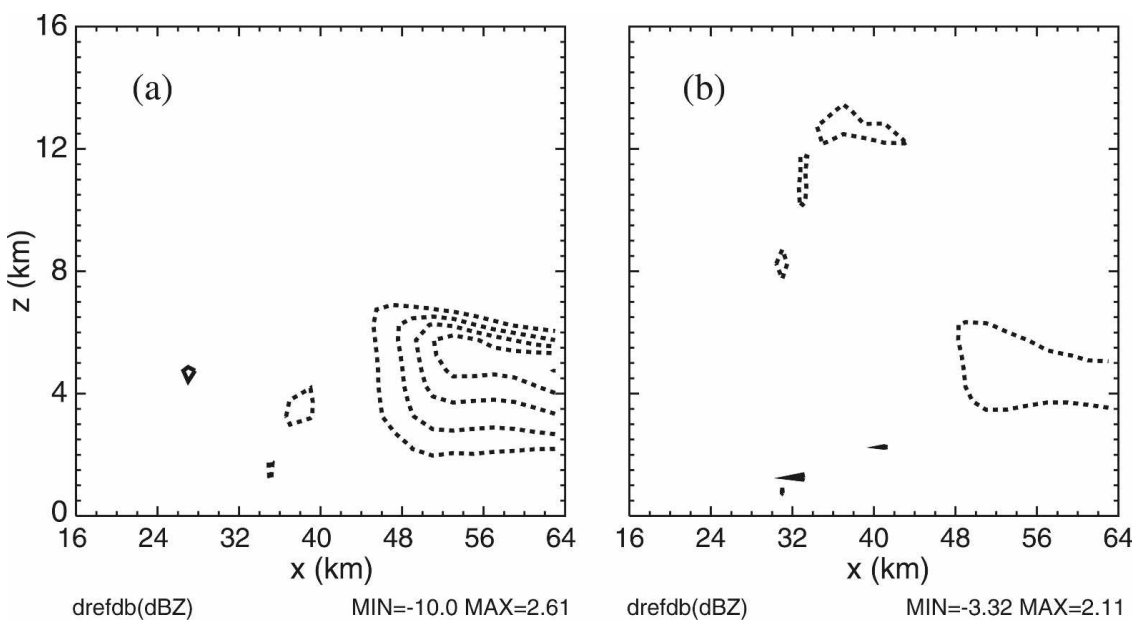

FIG. 7. Reflectivity differences between the sensitivity experiments and CNTL (sensitivity CNTL) at contour levels of $\pm 26, \pm 22, \pm 18, \pm 14, \pm 10, \pm 8, \pm 6, \pm 4, \pm 2$, and $0 \mathrm{dBZ}$ [solid (dashed) contours represent positive (negative) values] through the maximum updraft at $t=$ 70 min for forecast experiments (a) N0h43 $\rho_{h} 400$ with $n_{0 h}=4 \times 10^{3}\left(\mathrm{~m}^{-4}\right)$ and $\rho_{h}=400\left(\mathrm{~kg} \mathrm{~m}^{-3}\right)$ and (b) N0h43N0s55 $\rho_{h} 400$ with $n_{0 h}=4 \times 10^{3}\left(\mathrm{~m}^{-4}\right), n_{0 s}=5 \times 10^{5}\left(\mathrm{~m}^{-4}\right)$, and $\rho_{h}=400\left(\mathrm{~kg} \mathrm{~m}^{-3}\right)$.

zero deviation point (Fig. 3c). This as well as the smaller sensitivity of $V_{r}$ to the five parameters, as indicated by the smaller slopes of the response functions (Figs. 3a,b), suggests that $V_{r}$ observations are not as useful in estimating the microphysical parameters.

From the flatness of the response functions $J_{Z}$ near the bottom of their curves or from how far the local minima of $J_{Z}$ are located away from the true values (Fig. 3d), we can estimate how accurate the parameters might be estimated from reflectivity data. Figure $3 \mathrm{~d}$ shows that if the response functions are minimized, the error of the estimated $10 \log _{10}\left(n_{0 h}\right)$ is expected to vary within $[-1,1]$. The estimations of $n_{0 s}$ and $n_{0 r}$ may not be as accurate as that of $n_{0 h}$ due to the lower sensitivity of reflectivity around their true values. The error of the estimated $10_{10} \log \left(\rho_{h}\right)$ is expected to vary within $[-0.5$, $0.5]$, while the error of the estimated $10_{10} \log \left(\rho_{s}\right)$ can be as large as \pm 1 .

\section{b. Parameter identifiability as revealed by the correlation between model outputs of observations and microphysical parameters}

The uniqueness of the inverse problem for singleparameter estimation has been suggested by the shape of the response functions shown in Fig. 3. However, as the number of the parameters to be estimated increases, this may no longer be the case, as suggested in the previous subsection. Creating multidimensional response functions with respect to multiple parameters is expensive. An alternative way to examine parameter identifiability is to calculate the correlation coefficients between the parameters and the model output of the observed quantities, that is, covariance $\operatorname{cov}\left(P_{i}, y\right)$ normalized by the variances of $P_{i}$ and $y$, from the forecast ensemble. This is especially helpful for understanding the ensemble-based parameter estimation, because in this case the parameter is adjusted based on the covariances calculated from the ensemble.

To calculate the parameter model output correlations, several ensemble forecast experiments were performed. The initial ensemble was taken from the analysis ensemble of one of the CNTL assimilation experiments at $65 \mathrm{~min}$. The microphysical parameters were perturbed about their means individually or in different combinations in different experiments. Their true values were chosen to be their ensemble means, except for $\rho_{h}$ whose mean was set to be $700 \mathrm{~kg} \mathrm{~m}^{-3}$ to allow for both positive and negative perturbations. Here, we use $P_{i}=10 \log _{10}\left(p_{i}\right)$ in place of $p_{i}$ because the variation of $p_{i}$ can be more than an order of magnitude; the sampling of $p_{i}$ from a broad distribution can easily lead to unphysical negative values. The standard deviations of the parameter perturbations are roughly half of their largest deviations from their control values, that is, $1 / 2 \max \left(\left|\underline{P}_{i}-P_{\mathrm{ic}}\right|,\left|\bar{P}_{i}-P_{\mathrm{ic}}\right|\right)$. The correlation coefficients are calculated at $70 \mathrm{~min}$, or from 5-min forecasts, at observation points where simulated reflectivity data are greater than $0 \mathrm{dBZ}$.

Figures $8 \mathrm{a}-\mathrm{e}$ show the spatial structures of the correlation coefficients, at selected radar elevation levels, between each of the five microphysical parameters and the forecast reflectivity fields from the five ensemble 


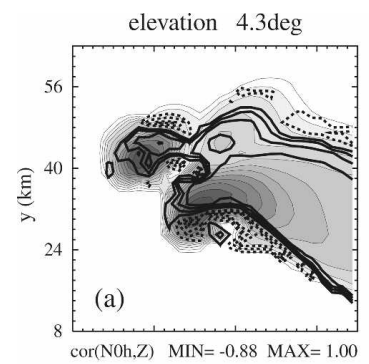

elevation $4.3 \mathrm{deg}$

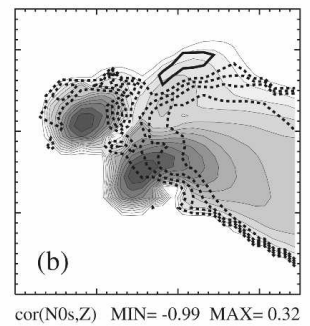

$\mathrm{n} 0 \mathrm{~h}=4 \times 10^{5}\left(\mathrm{~m}^{-4}\right)$

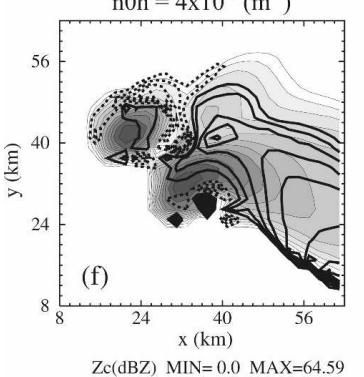

$\mathrm{n} 0 \mathrm{~s}=3 \times 10^{7}\left(\mathrm{~m}^{-4}\right)$

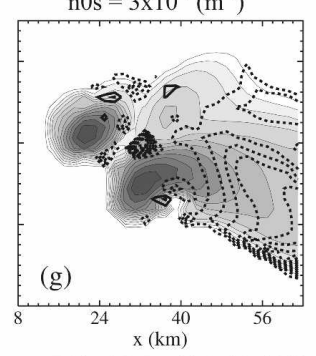

$\mathrm{Zc}(\mathrm{dBZ}) \mathrm{MIN}=0.0 \mathrm{MAX}=64.59$ elevation $0.5 \mathrm{deg}$

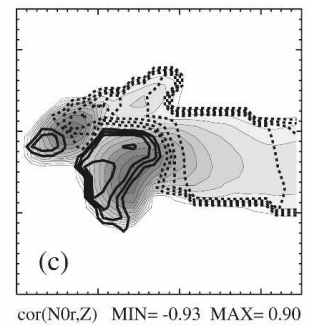

$\mathrm{n} 0 \mathrm{r}=8 \times 10^{6}\left(\mathrm{~m}^{-4}\right)$

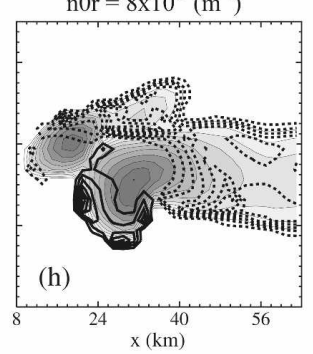

$\mathrm{Zc}(\mathrm{dBZ}) \mathrm{MIN}=0.0 \mathrm{MAX}=55.12$ $\mathrm{Zc}(\mathrm{dBZ}) \quad \mathrm{MIN}=0.0 \quad \mathrm{MAX}=55.12$
$\mathrm{cc}(\mathrm{dBZ})$
$\mathrm{MIN}=-16.98$

elevation $4.3 \mathrm{deg}$

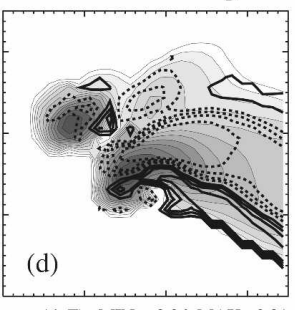

$\operatorname{cor}(\mathrm{rh}, \mathrm{Z}) \quad \mathrm{MIN}=-0.96 \mathrm{MAX}=0.91$ $\rho \mathrm{h}=400\left(\mathrm{~kg} \mathrm{~m}^{-3}\right)$

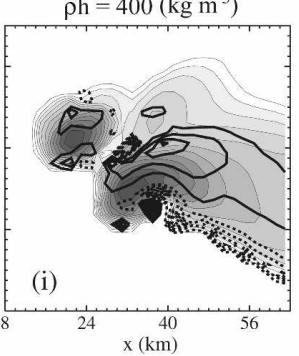
$\mathrm{Zc}(\mathrm{dBZ}) \mathrm{MIN}=0.0 \quad \mathrm{MAX}=64.59$
$\mathrm{Zc}(\mathrm{dBZ}) \mathrm{MIN}=-18.73 \quad \mathrm{MAX}=14.72$

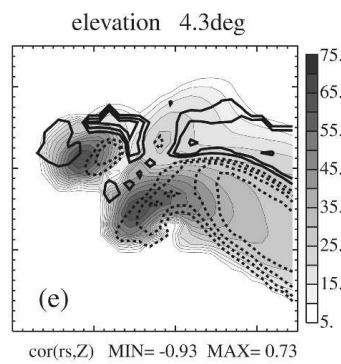

ps $=400\left(\mathrm{~kg} \mathrm{~m}^{-3}\right)$

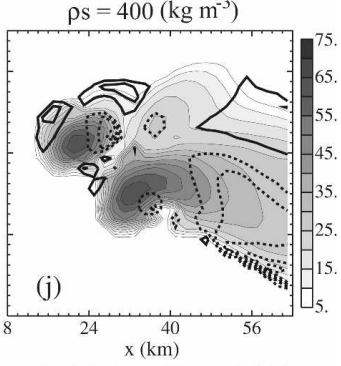

$\mathrm{Zc}(\mathrm{dBZ}) \quad \mathrm{MIN}=0.0 \quad \mathrm{MAX}=64.59$

FIG. 8. Correlations [solid (dashed) contours represent positive (negative) values at intervals of 0.2] between forecast reflectivity at observation points where simulated reflectivity data are greater than $0 \mathrm{dBZ}$, and logarithmically transformed (f) $n_{0 h}$, (g) $n_{0 s}$, (h) $n_{0 r}$, (i) $\rho_{h}$, and (j) $\rho_{s}$ estimated from members of single-parameter forecast ensemble Reflectivity difference [thick solid (dashed) contours represent positive (negative) values at intervals of $2 \mathrm{~dB}$ ] between forecast sensitivity experiments (a) N0h45, (b) N0s37, (c) N0r87, (d) $\rho_{h} 400$, and (e) $\rho_{s} 400$ and the CNTL forecast experiment at selected elevation levels at $t=70 \mathrm{~min}$. The shading and thin solid contours represent $Z$ from the CNTL forecast experiment.

forecast experiments that each perturbs only one parameter. To facilitate the understanding of the physical meanings of the correlations, the differences in $Z$ between each of the five sensitivity forecast experiments and the CNTL forecast experiment are shown in Figs. $8 \mathrm{f}-\mathrm{j}$. These forecast experiments were initialized from the ensemble mean analysis of a CNTL data assimilation experiment at $60 \mathrm{~min}$, which correspond to those shown in Fig. 5. The parameters were perturbed with the larger errors given in Table 2. The radar elevation levels are selected where large sensitivities in $Z$ are found.

It can be seen from Fig. 8 that in certain regions of the storm, where large sensitivities in reflectivity are found, in, for example, the anvil or anvil precipitation region, the microphysical parameters are highly correlated with reflectivity. The correlation patterns are consistent with the variations in reflectivity due to the changes in the parameters, as discussed earlier. For example, a larger $n_{0 r}$ results in smaller reflectivity at the low-level anvil precipitation region (Fig. 8h); therefore, $n_{0 r}$ should be negatively correlated with $Z$ there, which is actually found in Fig. 8c. A larger $n_{0 h}$ leads to larger reflectivity in the anvil (Fig. 8f). Consistently, positive correlations are found there. The correlations estimated from the ensemble members reveal unambiguous model responses to the errors in individual param- eters, suggesting again that the individual parameters can be estimated using reflectivity data.

Figure 9 shows that when all three intercept parameters are uncertain, we can still observe the right correlation patterns at the right locations. Among the three parameters, the correlation pattern for $n_{0 s}$ has more noticeable changes from the single-parameter case (Fig. 9b versus Fig. 8b) than the correlations for $n_{0 h}$ (Fig. 9a versus Fig. 8a) and $n_{0 r}$ (Fig. 9c versus Fig. $8 \mathrm{c})$. The correlation level for reflectivity, as indicated by the spatially averaged absolute correlation coefficients listed in Table 3, decreases by about $10 \%$ for $n_{0 h}$ (from 0.53 to 0.48 ) and $n_{0 r}$ (from 0.22 to 0.20 ), while the corresponding decrease for $n_{0 s}$ is about $36 \%$ (from 0.47 to 0.3 ). The correlation information suggests that the identifiability of $n_{0 s}$ is affect more by the presence of multiple parameter errors. We can also see from Table 3 that the correlation coefficients for radial velocity are always smaller than the corresponding coefficients for reflectivity, again indicating the less usefulness of radial velocity data for microphysical parameter estimation.

As the number of adjustable parameters further increases to five, the correlation level further decreases for all parameters (Table 3). At the selected elevation levels shown in Fig. 10, larger decreases in correlations are found for $n_{0 s}, \rho_{h}$, and $\rho_{s}$. Compared with the threeparameter case (Fig. 9c), very little change is found in 

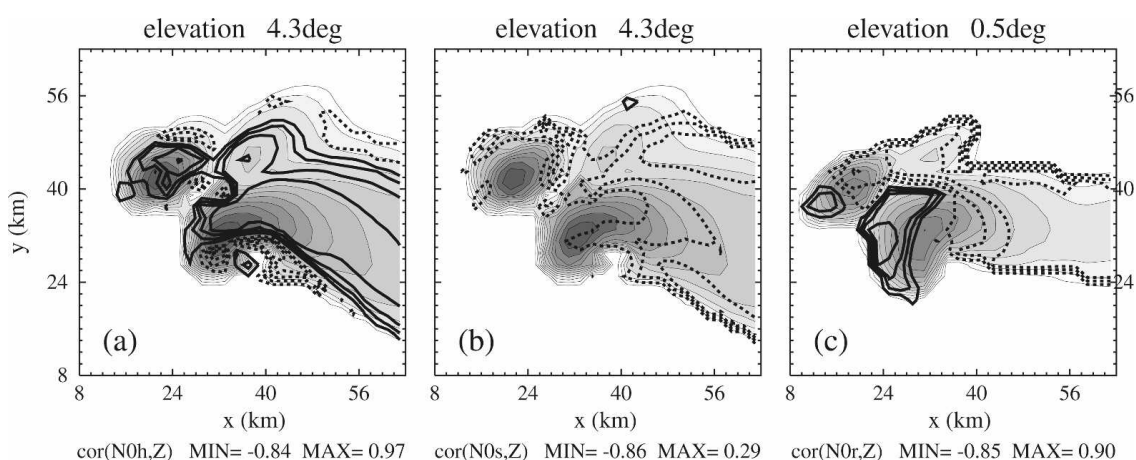

FIG. 9. Correlations [thick solid (dashed) contours represent positive (negative) values at intervals of 0.2] between reflectivity and logarithmically transformed (a) $n_{0 h}$, (b) $n_{0 s}$, (c) $n_{0 r}$, estimated from members of the three-parameter forecast ensemble at $t=70 \mathrm{~min}$. The shading and thin solid contours represent $Z$ from the control forecast experiment. Note that the elevation in (c) is $0.5^{\circ}$.

the correlations for $n_{0 r}$ (Fig. 10c) at the elevation level shown. Although the areas covered by significant correlations decrease for the five-parameter case, positive and negative correlations are found at similar locations as in the single-parameter cases. This suggests that it may still be possible to estimate the five parameters simultaneously, because the basic information contained in the correlation coefficients remains correct. However, with the correlations being generally weaker, there is a greater chance for errors in observations to cause instability in parameter estimation.

We also note that in some areas of the storm, model reflectivity output is not sensitive to the errors in the microphysical parameters, and in many areas the reflectivity-parameter correlations are rather weak, especially when more parameters are uncertain. The reflectivity data in those low-sensitivity-low-correlation regions will not be very useful for the microphysical parameter estimation.

\section{Summary and conclusions}

The possibility of estimating five fundamental microphysical parameters from radar observations is investi- gated by examining issues associated with parameter sensitivity and identifiability. These five parameters are the intercept parameters for rain, snow, and hail/ graupel and the bulk densities of hail/graupel and snow, which are usually prespecified constants in singlemoment bulk microphysics schemes and are involved in the definition of drop-particle size distributions. The identifiability of individual parameters is examined from two aspects: the sensitivity of the model response in terms of the observed quantities to the changes in the parameter values and the uniqueness of the inverse problem solution for parameter estimation.

Sensitivity analyses were carried out based on data assimilation and forecast experiments for a supercell thunderstorm case. To determine the model response to individual parameters, the parameters are varied within their observed ranges of uncertainty individually in the sensitivity experiments. A forecast response function, which measures the difference between the observations and the corresponding model forecast subjecting to the parameter error, was calculated for each of the sensitivity experiments. The response functions obtained from ensemble mean forecast within data assimilation cycles show concave shapes with

TABLE 3. Absolute values of correlations, averaged over observation points, at which $Z>0 \mathrm{~dB} Z$, at $t=70$ min, between forecast $Z$ or $V_{r}$ and one of the logarithmically transformed microphysical parameters as estimated from the forecast ensembles with different parameters perturbed from their true values, except $\rho_{s}$, which is perturbed from $700 \mathrm{~kg} \mathrm{~m}^{-3}$.

\begin{tabular}{|c|c|c|c|c|c|c|c|c|c|c|}
\hline \multirow[b]{2}{*}{ Experiment } & \multicolumn{2}{|c|}{$n_{0 h}$} & \multicolumn{2}{|c|}{$n_{0 s}$} & \multicolumn{2}{|c|}{$n_{0 r}$} & \multicolumn{2}{|c|}{$\rho_{h}$} & \multicolumn{2}{|c|}{$\rho_{s}$} \\
\hline & $Z$ & $V_{r}$ & $Z$ & $V_{r}$ & $Z$ & $V_{r}$ & $Z$ & $V_{r}$ & $Z$ & $V_{r}$ \\
\hline 1-para & 0.53 & 0.21 & 0.47 & 0.18 & 0.22 & 0.12 & 0.41 & 0.14 & 0.30 & 0.15 \\
\hline 2-para $\left(n_{0 h}, \rho_{h}\right)$ & 0.49 & 0.20 & & & & & 0.20 & 0.13 & & \\
\hline 2-para $\left(n_{0 s}, \rho_{s}\right)$ & & & 0.39 & 0.17 & & & & & 0.22 & 0.14 \\
\hline 3 -para $\left(n_{0 r}, n_{0 s}, n_{0 h}\right)$ & 0.48 & 0.20 & 0.30 & 0.17 & 0.20 & 0.12 & & & & \\
\hline 4-para $\left(n_{0 r}, n_{0 s}, n_{0 h}, \rho_{h}\right)$ & 0.45 & 0.20 & 0.27 & 0.17 & 0.19 & 0.12 & 0.21 & 0.13 & & \\
\hline 5-para (all five) & 0.44 & 0.18 & 0.23 & 0.16 & 0.17 & 0.11 & 0.19 & 0.12 & 0.18 & 0.14 \\
\hline
\end{tabular}



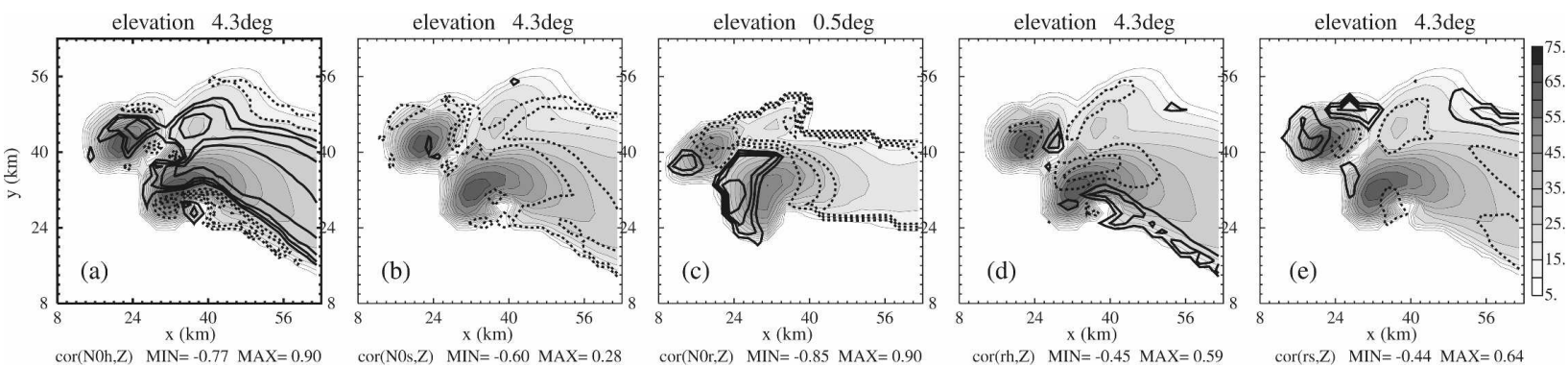

FIG. 10. As in Fig. 9, but the correlation coefficients are estimated from the members of the five-parameter forecast ensemble at

$$
t=70 \mathrm{~min} \text {. }
$$

unique minima. This indicates a high probability of being able to successfully estimate microphysical parameters when error only exists in one parameter. The results also show that the forecast reflectivity is more sensitive to the microphysical parameters than the model radial velocity; reflectivity, therefore, is preferred over radial velocity for microphysical parameter estimation.

The model response time to errors in individual parameters were estimated from the time evolution of response function ratios between the parameter-errorcontaining and parameter-error-free forecasts. The time that the ratio peaks is found to be between 5 and 15 min, suggesting rather fast model response to the parameter error and also the need to use frequent assimilation cycles of 5-10 min long to achieve effective parameter estimation before the model response becomes overwhelmed by the forecast error due to initial condition error.

The identifiability of the microphysical parameters is further evaluated using their correlations with the model output of radar observations based on ensemble forecasts. Each individual parameter is highly correlated with radar reflectivity at certain regions of the storm. The physical meanings of the correlations between the microphysical parameters and radar reflectivity can be understood by examining the sensitivities in microphysical fields. As the number of uncertain parameters increases, both the level and area coverage of significant correlation decrease, suggesting increased difficulties in estimating multiple parameters simultaneously.

In Part II, the details of the simultaneous estimation of the microphysical parameters and model state variables using the EnSRF algorithm from radar data will be presented. The sensitivity analysis and parameter identifiability discussed here will guide us with the experiment design and help us understand the results of estimation. The parameter identifiability issue will be further discussed there based on the estimation results.
Acknowledgments. This work was primarily supported by NSF Grants ATM-0129892 and ATM0530814. M. Xue was further supported by NSF Grants EEC-0313747, ATM-0331594, and ATM-0608168, and by grants from Chinese Academy of Sciences (2004-2-7) and Chinese Natural Science Foundation (40620120437). Comments and suggestions by anonymous reviewers significantly improved our paper.

\section{REFERENCES}

Aksoy, A., F. Zhang, and J. W. Nielsen-Gammon, 2006: Ensemble-based simultaneous state and parameter estimation in a two-dimensional sea-breeze model. Mon. Wea. Rev., 134, 2951-2970.

Anderson, J. L., 2001: An ensemble adjustment Kalman filter for data assimilation. Mon. Wea. Rev., 129, 2884-2903.

Bishop, C. H., B. J. Etherton, and S. J. Majumdar, 2001: Adaptive sampling with the ensemble transform Kalman filter. Part I: Theoretical aspects. Mon. Wea. Rev., 129, 420-436.

Braham, R. R., Jr., 1990: Snow particle size spectra in lake effect snows. J. Appl. Meteor., 29, 200-207.

Brandes, E. A., K. Ikeda, G. Zhang, M. Schönhuber, and R. M. Rasmussen, 2007: A statistical and physical description of hydrometeor distributions in Colorado snowstorms using a video disdrometer. J. Appl. Meteor. Climatol., 46, 634-650.

Burgers, G., P. J. van Leeuwen, and G. Evensen, 1998: Analysis scheme in the ensemble Kalman filter. Mon. Wea. Rev., 126, 1719-1724.

Caya, A., J. Sun, and C. Snyder, 2005: A comparison between the 4DVAR and the ensemble Kalman filter techniques for radar data assimilation. Mon. Wea. Rev., 133, 3081-3094.

Chavent, G., 1974: Identification of functional parameter in partial differential equations. Identification of Parameters in Distributed Systems: A Symposium of the American Automatic Control Council, R. E. Goodson and M. Polis, Eds., American Society of Mechanical Engineers, 31-48.

, 1979: Identification of distributed parameter systems: About the output least square method, its implementation, and identifiability. Identification and System Parameter Estimation: Proceedings of the Fifth IFAC Symposium, Vol. 1, R. Iserman, Ed., Pergamon Press, 85-97.

Cifelli, R., C. R. Williams, D. K. Rajopadhyaya, S. K. Avery, K. S. Gage, and P. T. May, 2000: Drop-size distribution characteristics in tropical mesoscale convective systems. J. Appl. Meteor., 39, 760-777. 
Cohard, J.-M., and J.-P. Pinty, 2000: A comprehensive twomoment warm microphysical bulk scheme. I: Description and tests. Quart. J. Roy. Meteor. Soc., 126, 1815-1842.

Courtier, P., and O. Talagrand, 1987: Variational assimilation of meteorological observations with the adjoint equation. Part II: Numerical results. Quart. J. Roy. Meteor. Soc., 113, 13291347.

Crook, N. A., 1996: Sensitivity of moist convection forced by boundary layer processes to low-level thermodynamic fields. Mon. Wea. Rev., 124, 1767-1785.

Dowell, D. C., F. Zhang, L. J. Wicker, C. Snyder, and N. A. Crook, 2004: Wind and temperature retrievals in the 17 May 1981 Arcadia, Oklahoma, supercell: Ensemble Kalman filter experiments. Mon. Wea. Rev., 132, 1982-2005.

El-Magd, A., V. Chandrasekar, V. N. Bringi, and W. Strapp, 2000: Multiparameter radar and in situ aircraft observation of graupel and hail. IEEE Trans. Geosci. Remote Sens., 38, 570-578.

Evensen, G., 1994: Sequential data assimilation with a nonlinear quasi-geostrophic model using Monte Carlo methods to forecast error statistics. J. Geophys. Res., 99 (C5), 10 143-10 162.

_ 2003: The ensemble Kalman filter: Theoretical formulation and practical implementation. Ocean Dyn., 53, 343-367.

— eter data for the Agulhas Current using the ensemble Kalman filter with a quasigeostrophic model. Mon. Wea. Rev., 124, 85-96.

Ferrier, B. S., 1994: A double-moment multiple-phase four-class bulk ice scheme. Part I: Description. J. Atmos. Sci., 51, 249280.

_, W.-K. Tao, and J. Simpson, 1995: A double-moment multiple-phase four-class bulk ice scheme. Part II: Simulations of convective storms in different large-scale environments and comparisons with other bulk parameterizations. J. Atmos. Sci., 52, 1001-1033.

Gaspari, G., and S. E. Cohn, 1999: Construction of correlation functions in two and three dimensions. Quart. J. Roy. Meteor. Soc., 125, 723-757.

Gilmore, M. S., J. M. Straka, and E. N. Rasmussen, 2004: Precipitation uncertainty due to variations in precipitation particle parameters within a simple microphysics scheme. Mon. Wea. Rev., 132, 2610-2627.

Gunn, K. L. S., and J. S. Marshall, 1958: The distribution with size of aggregate snowflakes. J. Meteor., 15, 452-461.

Houtekamer, P. L., and H. L. Mitchell, 1998: Data assimilation using an ensemble Kalman filter technique. Mon. Wea. Rev., 126, 796-811.

Houze, R. A., Jr., P. V. Hobbs, P. H. Herzegh, and D. B. Parsons, 1979: Size distributions of precipitation particles in frontal clouds. J. Atmos. Sci., 36, 156-162.

— C.-P. Cheng, C. A. Leary, and J. F. Gamache, 1980: Diagnosis of cloud mass and heat fluxes from radar and synoptic data. J. Atmos. Sci., 37, 754-773.

Joss, J., and A. Waldvogel, 1969: Raindrop size distribution and sampling size errors. J. Atmos. Sci., 26, 566-569.

Kitamura, S., and S. Nakagiri, 1977: Identifiability of spatiallyvarying and constant parameters in distributed systems of parabolic type. SIAM J. Control Optim., 15, 785-802.

Le Dimet, F. X., and O. Talagrand, 1986: Variational algorithms for analysis and assimilation of meteorological observations: Theoretical aspects. Tellus, 38A, 97-110.

Lin, Y.-L., R. D. Farley, and H. D. Orville, 1983: Bulk parameterization of the snow field in a cloud model. J. Climate Appl. Meteor., 22, 1065-1092.
Lo, K. K., and R. E. Passarelli Jr., 1982: The growth of snow in winter storms: An airborne observational study. J. Atmos. Sci., 39, 697-706.

Lord, S. J., and Coauthors, 2001: Observing system simulation experiments for NPOESS. Preprints, Fifth Symp. on Integrated Observing Systems, Albuquerque, NM, Amer. Meteor. Soc., 168-173.

McCumber, M., W.-K. Tao, J. Simpson, R. Penc, and S.-T. Soong, 1991: Comparison of ice-phase microphysical parameterization schemes using numerical simulations of tropical convection. J. Appl. Meteor., 30, 985-1004.

McFarquhar, G. M., and R. A. Black, 2004: Observations of particle size and phase in tropical cyclones: Implications for mesoscale modeling of microphysical processes. J. Atmos. Sci., 61, 422-439.

Meyers, M. P., R. L. Walko, J. Y. Harrington, and W. R. Cotton, 1997: New RAMS cloud microphysics parameterization. Part II: The two-moment scheme. Atmos. Res., 45, 3-39.

Milbrandt, J. A., and M. K. Yau, 2005: A multimoment bulk microphysics parameterization. Part II: A proposed threemoment closure and scheme description. J. Atmos. Sci., 62, 3065-3081.

Mitchell, D. L., 1988: Evolution of snow-size spectra in cyclonic storms. Part I: Snow growth by vapor deposition and aggregation. J. Atmos. Sci., 45, 3431-3451.

Murakami, M., 1990: Numerical modeling of dynamical and microphysical evolution of an isolated convective cloud-The 19 July 1981 CCOPE cloud. J. Meteor. Soc. Japan, 68, 107127.

Navon, I. M., 1998: Practical and theoretical aspects of adjoint parameter estimation and identifiability in meteorology and oceanography. Dyn. Atmos. Oceans, 27, 55-79.

Passarelli, R. E. J., 1978: Theoretical and observational study of snow-size spectra and snowflake aggregation efficiencies. $J$. Atmos. Sci., 35, 882-889.

Pruppacher, H. R., and J. D. Klett, 1978: Microphysics of Clouds and Precipitation. Reidel, 714 pp.

Rutledge, S. A., and P. V. Hobbs, 1983: The mesoscale and microscale structure and organization of clouds and precipitation in midlatitude cyclones. VIII: A model for the "seederfeeder" process in warm-frontal rainbands. J. Atmos. Sci., 40, 1185-1206.

_ and - 1984: The mesoscale and microscale structure and organization of clouds and precipitation in midlatitude cyclones. XII: A diagnostic modeling study of precipitation development in narrow cold-frontal rainbands. J. Atmos. Sci., 41, 2949-2972.

Smith, P. L., Jr., C. G. Myers, and H. D. Orville, 1975: Radar reflectivity factor calculations in numerical cloud models using bulk parameterization of precipitation. J. Appl. Meteor., 14, 1156-1165.

Snyder, C., and F. Zhang, 2003: Assimilation of simulated Doppler radar observations with an ensemble Kalman filter. Mon. Wea. Rev., 131, 1663-1677.

Straka, J. M., and E. R. Mansell, 2005: A bulk microphysics parameterization with multiple ice precipitation categories. $J$. Appl. Meteor., 44, 445-466.

Sun, N.-Z., and W.-G. Yeh, 1990: Coupled inverse problems in groundwater modeling 2 . Identifiability and experimental design. Water Resour. Res., 26, 2527-2540.

Tippett, M. K., J. L. Anderson, C. H. Bishop, T. M. Hamill, and J. S. Whitaker, 2003: Ensemble square root filters. Mon. Wea. Rev., 131, 1485-1490. 
Tokay, A., and D. A. Short, 1996: Evidence from tropical raindrop spectra of the origin of rain from stratiform versus convective clouds. J. Appl. Meteor., 35, 355-371.

,-- , and O. W. Thiele, 1995: Convective vs. stratiform rain over Kapingamarangi atoll during TOGA COARE: Evidence from raindrop spectra. Preprints, Conf. on Cloud Physics, Dallas, TX, Amer. Meteor. Soc., 252-257.

Tong, M., 2006: Ensemble Kalman filter assimilation of Doppler radar data for the initialization and prediction of convective storms. Ph.D. dissertation, University of Oklahoma, 243 pp. , and M. Xue, 2005: Ensemble Kalman filter assimilation of Doppler radar data with a compressible nonhydrostatic model: OSS experiments. Mon. Wea. Rev., 133, 1789-1807.

$\longrightarrow$, and - 2008: Simultaneous estimation of microphysical parameters and atmospheric state with simulated radar data and ensemble square root Kalman filter. Part II: Parameter estimation experiments. Mon. Wea. Rev., 136, 1649-1668.

van den Heever, S. C., and W. R. Cotton, 2004: The impact of hail size on simulated supercell storms. J. Atmos. Sci., 61, 15961609.

Waldvogel, A., 1974: The $N_{0}$ jump of raindrop spectra. J. Atmos. Sci., 31, 1067-1078.

Whitaker, J. S., and T. M. Hamill, 2002: Ensemble data assimilation without perturbed observations. Mon. Wea. Rev., 130, 1913-1924.

Xue, M., K. K. Droegemeier, and V. Wong, 2000: The Advanced Regional Prediction System (ARPS) - A multiscale nonhydrostatic atmospheric simulation and prediction tool. Part I:
Model dynamics and verification. Meteor. Atmos. Phys., 75, 161-193.

, and Coauthors, 2001: The Advanced Regional Prediction System (ARPS) - A multiscale nonhydrostatic atmospheric simulation and prediction tool. Part II: Model physics and applications. Meteor. Atmos. Phys., 76, 143-166.

, D.-H. Wang, J.-D. Gao, K. Brewster, and K. K. Droegemeier, 2003: The Advanced Regional Prediction System (ARPS), storm-scale numerical weather prediction and data assimilation. Meteor. Atmos. Phys., 82, 139-170.

—, M. Tong, and K. K. Droegemeier, 2006: An OSSE framework based on the ensemble square root Kalman filter for evaluating the impact of data from radar networks on thunderstorm analysis and forecasting. J. Atmos. Oceanic Technol., 23, 46-66.

Yakowitz, S., and L. Duckstein, 1980: Instability in aquifer identification: Theory and case studies. Water Resour. Res., 16, 1045-1064.

Yeh, W.-G., 1986: Review of parameter identification procedures in groundwater hydrology: The inverse problem. Water Resour. Res., 22, 95-108.

Zhang, F., C. Snyder, and J. Sun, 2004: Impacts of initial estimate and observation availability on convective-scale data assimilation with an ensemble Kalman filter. Mon. Wea. Rev., 132, $1238-1253$.

Ziegler, C. L., 1985: Retrieval of thermal and microphysical variables in observed convective storms. Part I: Model development and preliminary testing. J. Atmos. Sci., 42, 1487-1509. 